\title{
A FAR-IR VIEW OF THE STARBURST-DRIVEN SUPERWIND IN NGC 2146
}

\author{
K. Kreckel ${ }^{1}$, L. Armus ${ }^{2}$, B. Groves ${ }^{1}$, M. Lyubenova $^{1}$, T. Díaz-Santos ${ }^{2}$, E. Schinnerer ${ }^{1}$, P. Appleton $^{3}$, K. V. Croxall ${ }^{4}$, \\ D. A. Dale ${ }^{5}$, L. K. Hunt ${ }^{6}$, P. Beirão ${ }^{7}$, A. D. Bolatto ${ }^{8,9,18}$, D. Calzetti ${ }^{10}$, J. Donovan Meyer ${ }^{11}$, B. T. Draine ${ }^{12}$, \\ J. Hinz ${ }^{13,14}$, R. C. Kennicutt ${ }^{15}$, S. MeidT ${ }^{1}$, E. J. Murphy ${ }^{16}$, J. D. T. Smith ${ }^{17}$, F. S. TABAtabaei ${ }^{1}$, And F. Walter ${ }^{1}$ \\ ${ }^{1}$ Max Planck Institut für Astronomie, Königstuhl 17, D-69117 Heidelberg, Germany; kreckel@ mpia.de \\ ${ }^{2}$ Spitzer Science Center, California Institute of Technology, MC 314-6, Pasadena, CA 91125, USA \\ ${ }^{3}$ NASA Herschel Science Center, IPAC, California Institute of Technology, Pasadena, CA 91125, USA \\ ${ }^{4}$ Department of Astronomy, The Ohio State University, 140 West 18th Avenue, Columbus, OH 43210, USA \\ ${ }^{5}$ Department of Physics and Astronomy, University of Wyoming, Laramie, WY 82071, USA \\ ${ }^{6}$ INAF-Osservatorio Astrosico di Arcetri, Largo E. Fermi 5, I-50125 Firenze, Italy \\ ${ }^{7}$ Observatoire de Paris, 61 avenue de l'Observatoire, F-75014 Paris, France \\ ${ }^{8}$ Department of Astronomy and Joint Space-Science Institute, University of Maryland, College Park, MD, USA \\ ${ }^{9}$ Max-Planck Institute for Astronomy, D-69117 Heidelberg, Germany \\ ${ }^{10}$ Department of Astronomy, University of Massachusetts, Amherst, MA 01003, USA \\ ${ }^{11}$ National Radio Astronomy Observatory, Charlottesville, VA 22901, USA \\ 12 Princeton University Observatory, Peyton Hall, Princeton, NJ 08544-1001, USA \\ ${ }^{13}$ Steward Observatory, University of Arizona, Tucson, AZ 85721, USA \\ ${ }^{14}$ MMT Observatory, Tucson, AZ 85721, USA \\ ${ }^{15}$ Institute of Astronomy, University of Cambridge, Madingley Road, Cambridge CB3 OHA, UK \\ ${ }^{16}$ Infrared Processing and Analysis Center, California Institute of Technology, MC 220-6, Pasadena, CA 91125, USA \\ ${ }^{17}$ Department of Physics and Astronomy, University of Toledo, Toledo, OH 43606, USA \\ Received 2014 February 19; accepted 2014 May 4; published 2014 June 30
}

\begin{abstract}
NGC 2146, a nearby luminous infrared galaxy, presents evidence for outflows along the disk minor axis in all gas phases (ionized, neutral atomic, and molecular). We present an analysis of the multi-phase, starburst-driven superwind in the central $5 \mathrm{kpc}$ as traced in spatially resolved spectral line observations, using far-IR Herschel PACS spectroscopy, to probe the effects on the atomic and ionized gas, and optical integral field spectroscopy to examine the ionized gas through diagnostic line ratios. We observe an increased $\sim 250 \mathrm{~km} \mathrm{~s}^{-1}$ velocity dispersion in the [O I] $63 \mu \mathrm{m},[\mathrm{O} \mathrm{III}] 88 \mu \mathrm{m},[\mathrm{N}$ II $] 122 \mu \mathrm{m}$, and [C II] $158 \mu \mathrm{m}$ fine-structure lines that is spatially coincident with high excitation gas above and below the disk. We model this with a slow $\sim 200 \mathrm{~km} \mathrm{~s}^{-1}$ shock and trace the superwind to the edge of our field of view $2.5 \mathrm{kpc}$ above the disk. We present new SOFIA $37 \mu \mathrm{m}$ observations to explore the warm dust distribution, and detect no clear dust entrainment in the outflow. The stellar kinematics appear decoupled from the regular disk rotation seen in all gas phases, consistent with a recent merger event disrupting the system. We consider the role of the superwind in the evolution of NGC 2146 and speculate on the evolutionary future of the system. Our observations of NGC 2146 in the far-IR allow an unobscured view of the wind, crucial for tracing the superwind to the launching region at the disk center, and provide a local analog for future ALMA observations of outflows in high-redshift systems.
\end{abstract}

Key words: dust, extinction - galaxies: individual (NGC 2146) - galaxies: starburst - infrared: ISM ISM: kinematics and dynamics

Online-only material: color figures

\section{INTRODUCTION}

Galaxy outflows driven by star formation and active galactic nuclei (AGNs) allow redistribution of energy and metals within the interstellar medium (Veilleux et al. 2005) and have the potential to strongly affect galaxy evolution through quenching of star formation (Kormendy et al. 2009). They are a common feature in luminous infrared galaxies (LIRGs; Rupke et al. 2005), which are observed to increasingly dominate the star formation rate (SFR) density at higher redshifts up to $z \sim 2$ (Le Floc'h et al. 2005; Pérez-González et al. 2005).

NGC 2146 is one of the closest (17.2 Mpc, Tully 1988) infrared luminous $\left(L_{\mathrm{IR}}=1.2 \times 10^{11} L_{\odot}\right.$; Sanders et al. 2003) galaxies. With a stellar mass of $2 \times 10^{10} M_{\odot}$ (Skibba et al. 2011) and morphological type SB(s)ab pec (de Vaucouleurs et al. 1991), it displays a disturbed optical morphology due to a merger and a bright central bulge, extended irregular spiral arms, and

\footnotetext{
18 Visiting Humboldt Fellow.
}

deep dust lane features (Greve et al. 2006). H I imaging reveals an extremely extended $(\sim 200 \mathrm{kpc})$ tail (Fisher \& Tully 1976; Taramopoulos et al. 2001). NGC 2146 has a well established superwind along the minor axis that has been detected in X-rays and optical emission lines from ionized atomic gas that show evidence for shock excitation (Armus et al. 1995; Greve et al. 2000) as well as in a molecular gas outflow and superbubbles (Tsai et al. 2009). There is no evidence for AGN activity in the optical (Ho et al. 1997) or in mid-IR spectra (BernardSalas et al. 2009; Petric et al. 2011), though it may host a lowluminosity AGN based on its compact nuclear X-ray emission (Inui et al. 2005). As it exhibits a relatively high SFR of $7.9 M_{\odot}$ year $^{-1}$ (Kennicutt et al. 2011), stellar winds and supernovae are thought to drive the superwind, similar to that observed in the starburst galaxy M82 (McCarthy et al. 1987; Armus et al. 1989; Heckman et al. 1990). However, as NGC 2146 is both larger and more massive compared to M82, its outflow exhibits a larger geometric scale and slower wind velocities (Greve et al. 2000). 


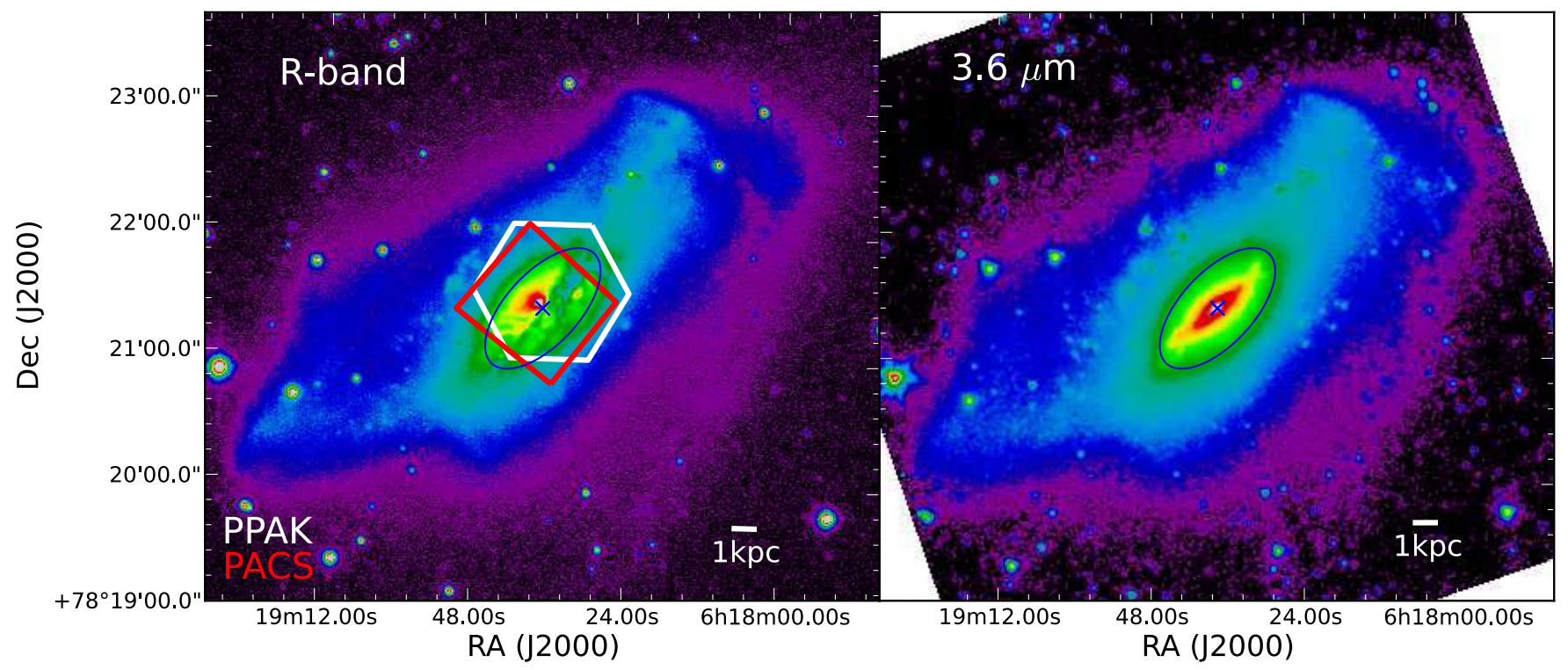

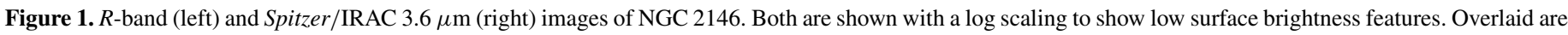

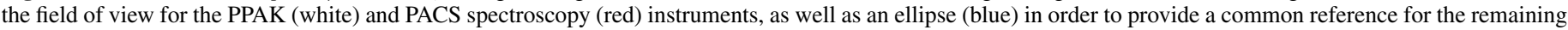

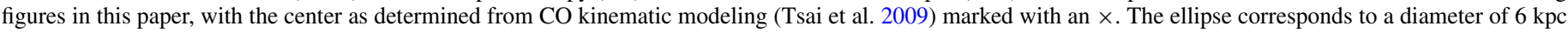
at the assumed distance.

(A color version of this figure is available in the online journal.)

Extensive analysis of the ionized and molecular gas kinematics have resulted in an understanding of the geometry of the system (Greve et al. 2000; Tsai et al. 2009). The disk is highly inclined at $63^{\circ}$ (della Ceca et al. 1999), with the near side of the disk in the southwest, making the southwest outflow behind and the northeast outflow in front of the disk. The outflow follows a conical morphology above and below the disk. The molecular gas outflow is seen through a breakout on the northeast side and two superbubbles, one to the southeast and another in projection along the major axis (Tsai et al. 2009). The combination of disk inclination angle and cone opening angle results in an unusual line-of-sight projection of the system, as diagramed in Greve et al. (2000) and Tsai et al. (2009). The far side of the cone to the north and the near side of the cone to the south are essentially perpendicular to the line of sight, making their velocities consistent with the systemic velocity. The other walls of the cone fall, in projection, over the bright central disk component and may not be easily seen.

Studies of nearby LIRGs and ULIRGs show they are generally interacting systems (Armus et al. 1987; Sanders et al. 1988; Murphy et al. 1996), and integral field spectroscopic surveys have revealed complex kinematics in multiple gas phases (Arribas et al. 2008; Alonso-Herrero et al. 2009, 2010). These kinematics develop along a merger sequence toward more elliptical or lenticular dynamics (Bellocchi et al. 2013). Many show evidence for shock ionization (Armus et al. 1989; MonrealIbero et al. 2010). However, optical studies are restricted by dust extinction, particularly along the galaxy disk and in the center where these winds are launched. Far-IR observations are able to penetrate these dusty regions, providing a direct view of the complete galactic wind. Such outflows have been observed within ULIRGs by molecular absorption against the nucleus (Fischer et al. 2010; Sturm et al. 2011; Veilleux et al. 2013; Spoon et al. 2013), but the possibility also exists to map outflow regions through the far-IR fine-structure emission lines.

We present here results from Herschel PACS spectroscopy, which reveal conical outflows in the atomic and ionized gas that can be traced back to the central region of the galaxy disk. We link this to new optical integral field spectroscopy that traces the ionized shock diagnostics over a broad region both above and below the galaxy disk. We also examine the warm and cold dust distribution through multi-wavelength farIR imaging. We present our observations in Section 2, and our results in Section 3. We discuss in Section 4 and conclude in Section 5.

\section{DATA}

We consider maps from the far-IR and optical lines over a $\sim 1^{\prime}$ field of view containing the central $\sim 5 \mathrm{kpc}$ of NGC 2146 (Figure 1). We also present new SOFIA $37 \mu \mathrm{m}$ images to further explore the warm dust distribution. All observations are centered on the bulge and exclude most of the extended disk. Due to the high inclination, they do contain spiral arm regions superimposed on the bulge, as seen by the deep dust lanes in the optical image. At the assumed distance of 17.2 Mpc (Tully 1988), 1" corresponds to $83 \mathrm{pc}$.

\subsection{Far-IR spectroscopy}

Using the PACS instrument (Poglitsch et al. 2010) on board Herschel (Pilbratt et al. 2010) we carried out far-IR spectral observations of the [OI] $63 \mu \mathrm{m}$, [O III] $88 \mu \mathrm{m}$, [N II] $122 \mu \mathrm{m}$, and [C II] $158 \mu \mathrm{m}$ fine-structure lines as part of the KINGFISH Open Time Key Program (Kennicutt et al. 2011). The species producing $[\mathrm{O} I I I],\left[\mathrm{N}_{\mathrm{II}}\right]$, and $\left[\mathrm{C}_{\mathrm{II}}\right]$ have ionization potentials of $35.12 \mathrm{eV}, 14.53 \mathrm{eV}$, and $11.26 \mathrm{eV}$, respectively. Observations were centered on the galactic nucleus of NGC 2146. All PACS spectral observations were obtained in the Un-Chopped Mapping mode and reduced using the Herschel Interactive Processing Environment version 11.2637. Reductions applied the standard spectral response functions and flat field corrections, flagged instrument artifacts and bad pixels, and subtracted the dark current. Transients caused by thermal instabilities were removed using a custom treatment designed for the KINGFISH 


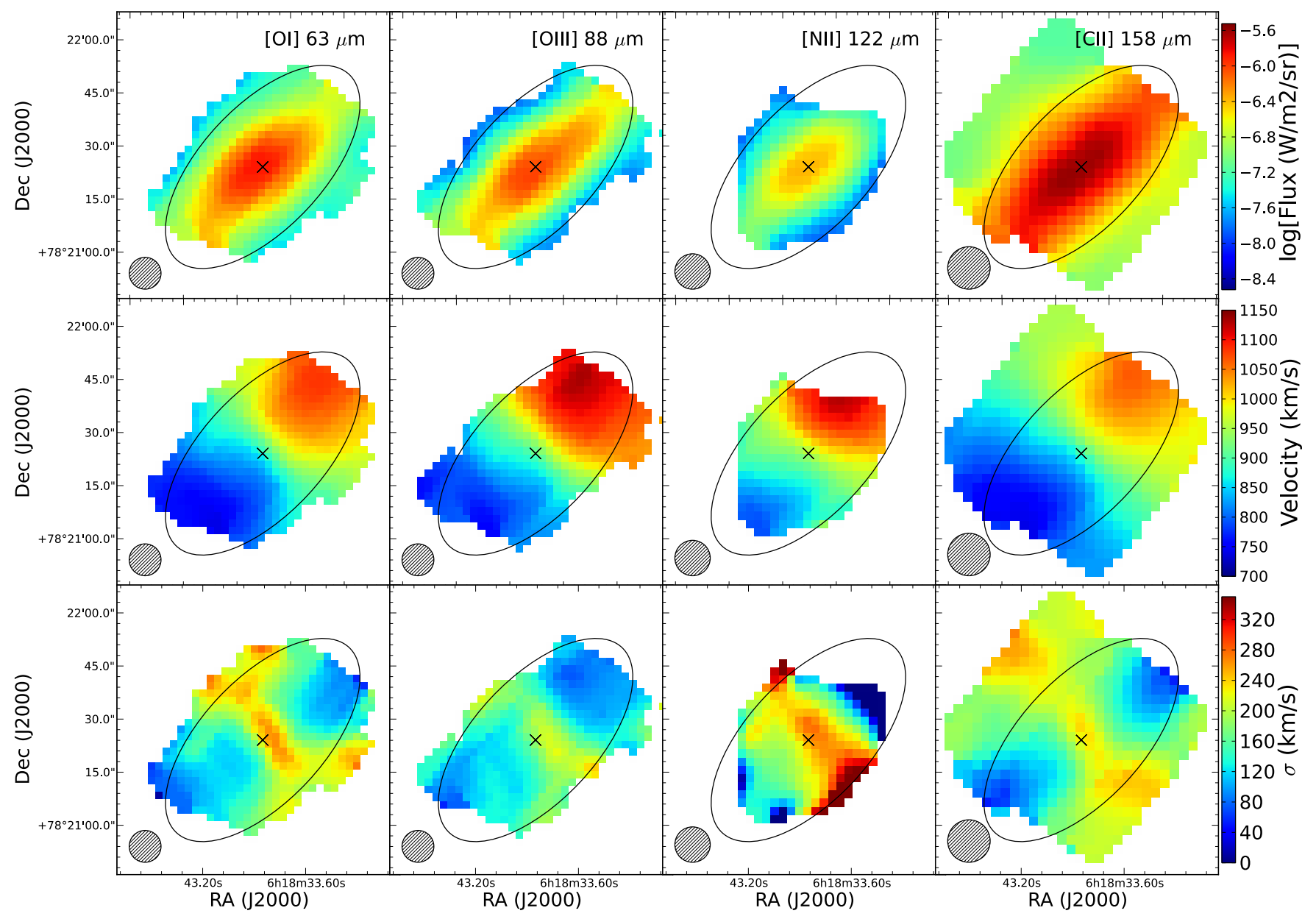

Figure 2. PACS spectroscopy line maps of (left to right) [O I] $63 \mu \mathrm{m}$, [O III] $88 \mu \mathrm{m}$, [N II] $122 \mu \mathrm{m}$, and [C II] $158 \mu \mathrm{m}$ of the intensity (top), velocity (middle), and deconvolved velocity dispersion (bottom). The ellipse and center match those shown in Figure 1. All lines are masked in regions where the signal to noise is less than five. Uncertainties in the velocity dispersion are $\sim 5 \%$ in the regions above and below the disk, and are much lower $(<1 \%)$ within the disk. [N II] has velocity dispersion peaking at $\sim 450 \mathrm{~km} \mathrm{~s}^{-1}$. The point-spread function for each map is indicated in the lower left corner, and the 6 kpc ellipse shown for reference.

(A color version of this figure is available in the online journal.)

Pipeline. Specific information on data reduction is contained in Croxall et al. (2013) and the KINGFISH Data Products Delivery (DR3) User's Guide. ${ }^{19}$ Flux maps were obtained by fitting single Gaussian profiles to each projected pixel. We extract line intensities and kinematics from a Gaussian fit to the line profile at each pixel in our line maps (Figure 2). Velocities are not corrected for inclination; however, given the high inclination of this galaxy, the corrections would be less than $10 \%$. Velocity dispersion measurements have been deconvolved to correct for the instrumental broadening that ranges from $100 \mathrm{~km} \mathrm{~s}^{-1}$ (at [O I]) to $180 \mathrm{~km} \mathrm{~s}^{-1}$ (at [C II]). Resulting line maps achieve an angular resolution that varies by wavelength between $9^{\prime \prime}-12^{\prime \prime}$. [N II] $205 \mu \mathrm{m}$ was also observed but is not significantly detected. Flux calibration of PACS data yield absolute flux uncertainties on the order of $15 \%$ with relative flux uncertainties between each Herschel pointing within a galaxy on the order of $\sim 10 \%$.

\subsection{Far-IR imaging}

SOFIA (Young et al. 2012) observations of NGC 2146 at $37.1 \mu \mathrm{m}$ using the FORCAST instrument (Herter et al. 2012) were carried out during four flights $(57,60,63$, and 64) as part of the Cycle 0 planID 81_0059 (PI: Armus). However, due to

\footnotetext{
19 http://herschel.esac.esa.int/UserProvidedDataProducts.shtml
}

adverse atmospheric conditions only data from three flights (57, 60, and 64) have been used in this paper. Level 3 images were retrieved from the Science Archive at the SOFIA Data Cycle System. ${ }^{20}$ Pre-processing of each image includes coadding of all chop-nod positions as well as correcting for spatial distortions. WCS is not available for Basic Science observations. Thus, the registeration of the coadded images was done by smoothing each image with a Gaussian kernel and finding its centroid. Images were stacked using weights proportional to $1 / \sigma^{2}$, where $\sigma$ is the standard deviation of the sky around the galaxy. Astrometry was applied by comparing the centroid to the MIPS (Rieke et al. 2004) $24 \mu \mathrm{m}$ image. Flux-calibration was performed using the multiplicative factor CALFACTR available in the header of each image. Final image resolution is diffraction-limited with a full width at half maximum of 3".5.

We also present archival Spitzer MIPS $24 \mu \mathrm{m}$ (Armus et al. 2009) and Herschel PACS $100 \mu \mathrm{m}, 160 \mu \mathrm{m}$, and SPIRE $250 \mu \mathrm{m}$ (Kennicutt et al. 2011) images for comparison.

\subsection{Optical IFU data}

Optical integral field unit (IFU) data were taken at the Calar Alto $3.5 \mathrm{~m}$ telescope using the PMAS instrument in PPAK

\footnotetext{
${ }^{20}$ https://dcs.sofia.usra.edu/
} 


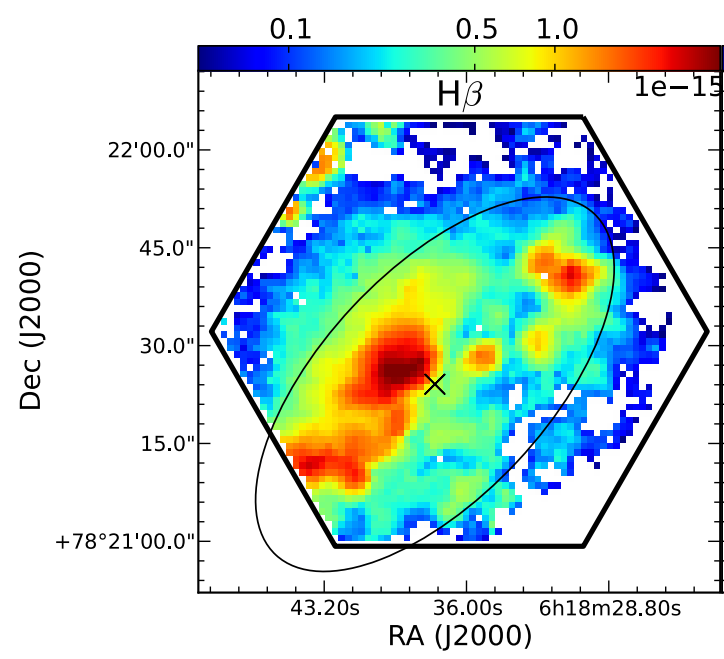

0.1 $0.5 \quad 1.0$ 1 5
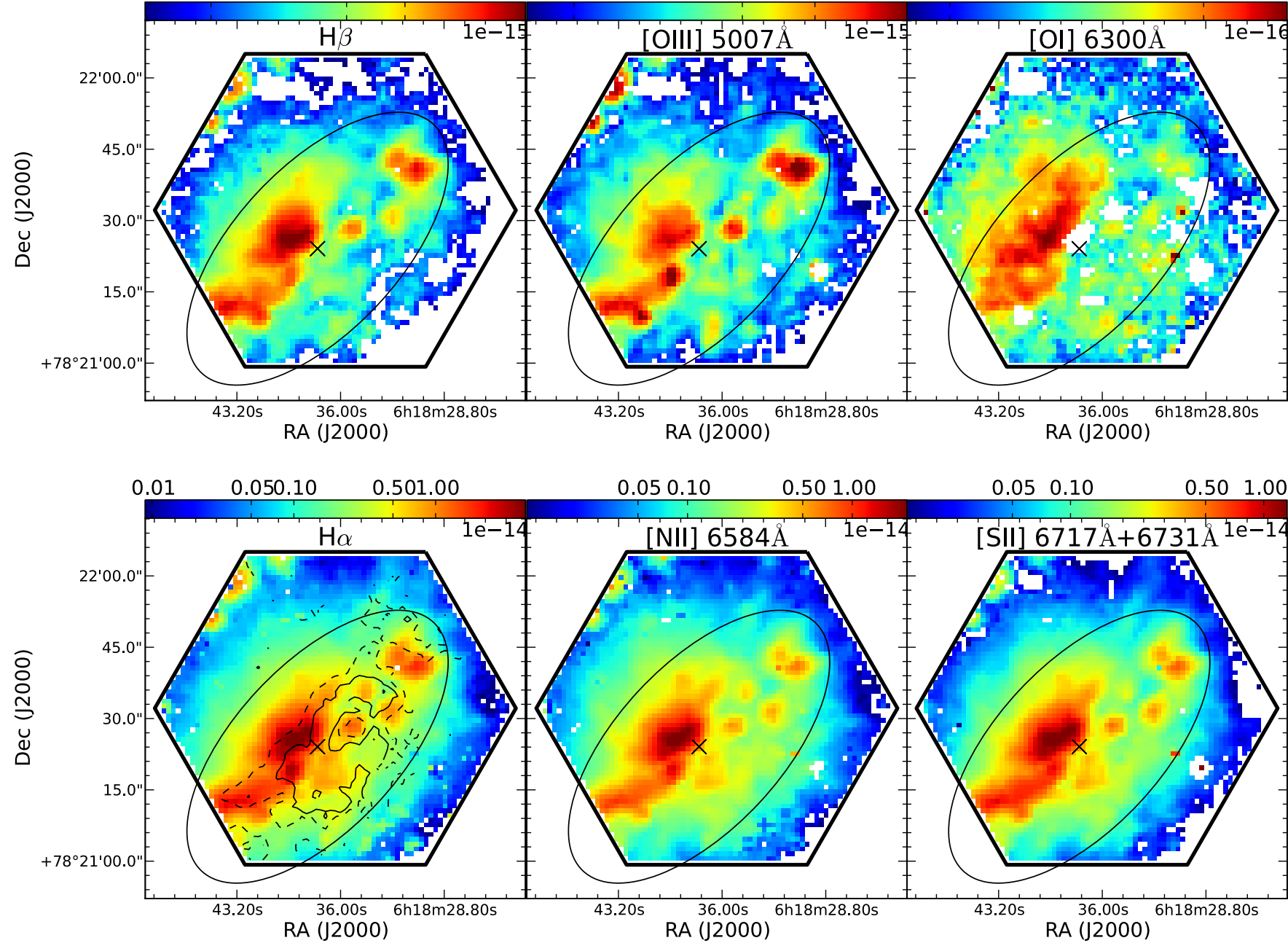

$0.050 .10 \quad 0.501 .00$

$0.05 \quad 0.10$

$0.50 \quad 1.00$

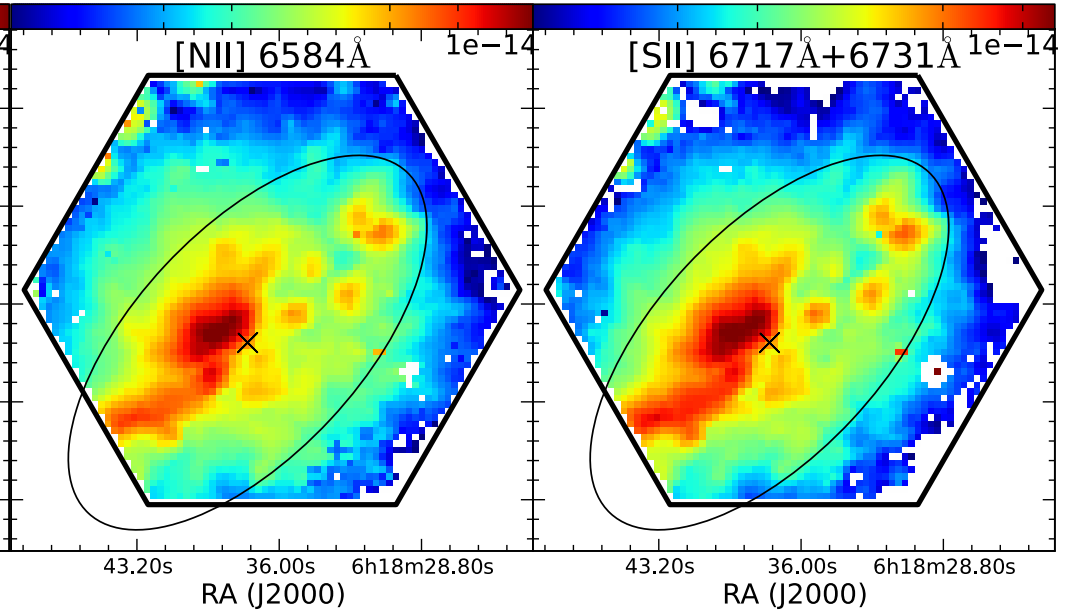

Figure 3. Line maps of $\mathrm{H} \beta$, [O III] $5007 \AA$, [O I] $6300 \AA$, $\mathrm{H} \alpha$, [N II] $6584 \AA$, and [S II] $6717 \AA+6731 \AA$ measured from the optical IFU data, where the color bars give the line flux for each map in units of erg s $\mathrm{cm}^{-2} \mathrm{arcsec}^{-2}$. In the $\mathrm{H} \alpha$ image we include contours at an extinction of $A_{\mathrm{V}}=3 \mathrm{mag}$ (dashed) and $A_{\mathrm{V}}=4 \mathrm{mag}$ ( $\mathrm{solid}$ ) to indicate where the dust becomes effectively opaque in the optical. The stellar disk and kinematic center (black) is marked for reference.

(A color version of this figure is available in the online journal.)

mode (Roth et al. 2005; Kelz et al. 2006), which consists of 331 fibers each 2".68 in diameter arranged in a hexagonal pattern to cover a $\sim 1^{\prime}$ field of view. Observations were performed using the V300 grating, which covers $3700-7000 \AA$ at $\sim 180 \mathrm{~km} \mathrm{~s}^{-1}$ velocity resolution, and dithered in three positions to recover the full flux and subsample the fiber size. The data reduction has been described in detail in Kreckel et al. (2013). All spectra were reduced using the p3d package (Sandin et al. 2010), and line fluxes were extracted using the Gandalf (Sarzi et al. 2006) and pPXF (Cappellari \& Emsellem 2004) software packages. We present line maps with a 2'.5 spatial resolution for the $\mathrm{H} \beta$, [O III] $5007 \AA$, [O I] $6300 \AA$, [N II] $6584 \AA, \mathrm{H} \alpha$, and both [S II] $6717 \AA$ and $6731 \AA$ A lines in Figure 3. The [N II] $6548 \AA$ line is also detected and is fit assuming a fixed ratio with the [N II] $6548 \AA$ line. The emission of [O I] is redshifted away from the skyline contamination.

When fitting the lines and continuum we tie the velocities and dispersion of the Balmer lines $(\mathrm{H} \alpha, \mathrm{H} \beta$, and where detected $\mathrm{H} \gamma$, $\mathrm{H} \delta$ ) and separately tie together the kinematics of the remaining nebular lines ([O III], [O I ], [N II], [S II]). Our requirement that tied lines exhibit matched dynamics is based on the assumption that they are coming from the same regions within the galaxy. Tying the lines improves the fit for low signal to noise lines, allowing us to measure the velocity centroid more accurately (Figure 4), despite the limited instrumental spectral resolution. We then obtain two independent measures of the kinematics from the Balmer and forbidden lines. As with the far-IR lines, velocities are not corrected for inclination. We measure the line centroid to within $20 \mathrm{~km} \mathrm{~s}^{-1}$ but do not resolve the velocity dispersion due to the large $\sim 180 \mathrm{~km} \mathrm{~s}^{-1}$ instrumental spectral resolution.

Simultaneous with our emission line fit using Gandalf we also fit with pPXF a combination of stellar templates based on the Tremonti et al. (2004) adaptation of the Bruzual \& Charlot (2003) simple stellar population (SSP) models. These cover a range of ages (5 Myr-11 Gyr) at solar metallicity. The wide wavelength range of our spectra and the fact that it includes the age-sensitive $4000 \AA$ break allows us to break the dust-age degeneracy (see also Kreckel et al. 2013). From this stellar template fit, which is masked to exclude emission lines and includes numerous stellar absorption features, we recover a stellar kinematic fit including both a velocity centroid and dispersion. Stellar velocities are accurate to $\sim 50 \mathrm{~km} \mathrm{~s}^{-1}$ and stellar velocity dispersions are largely unresolved at our spectral resolution. These measurements are only possible in regions not suffering from high dust extinction, therefore for a 


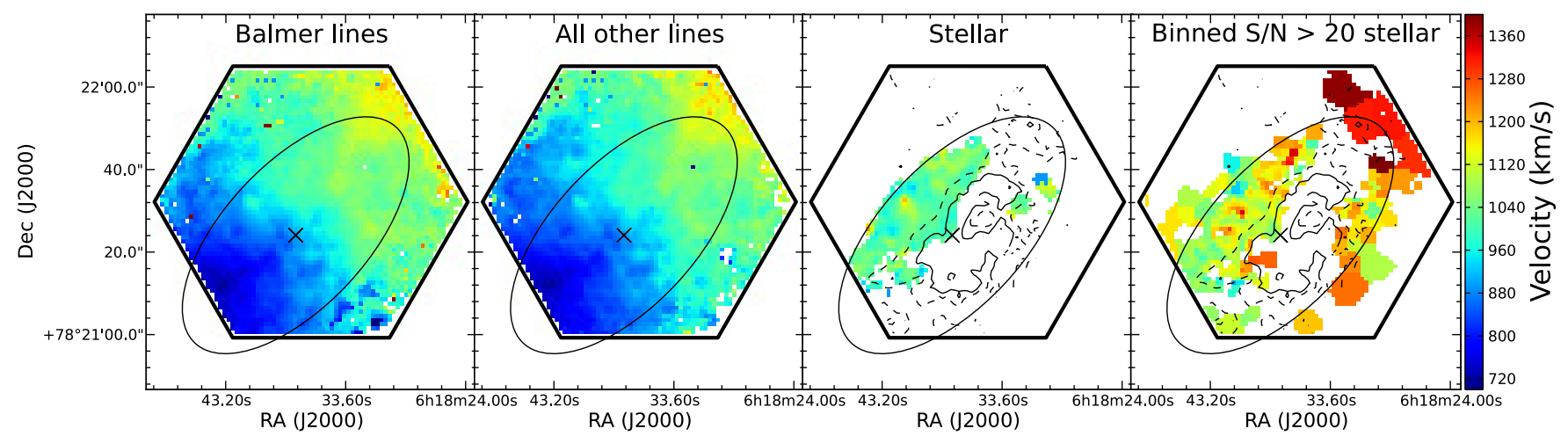

Figure 4. From left to right: velocities from fitting together all Balmer lines ( $\mathrm{H} \alpha, \mathrm{H} \beta$, and where detected $\mathrm{H} \gamma, \mathrm{H} \delta$ ), all other PPAK emission lines ([O III], [O I], [N II], [S II]), from the stellar absorption kinematics at full resolution and from the stellar absorption kinematics after binning pixels to a signal to noise $>20$. Emission line maps are masked at an amplitude over noise of 2 , stellar kinematics are masked at a signal to noise of 5 . Contours show a measured extinction of $A_{\mathrm{V}}=3$ mag (dashed) and $A_{\mathrm{V}}=4 \mathrm{mag}$ (solid) to indicate where the dust becomes effectively opaque in the stellar continuum. Velocity dispersions are not resolved due to the instrumental dispersion. The stellar disk and kinematic center (black) is marked for reference.

(A color version of this figure is available in the online journal.)

wide region located northeast of the dust lane we obtain robust solutions.

Voronoi binning (Cappellari \& Copin 2003) of neighboring spectra to ensure a minimum signal to noise of 20 per region in the stellar continuum recovers detections across much of the stellar disk (Figure 4). For these binned regions we fit a nonlinear combination of 330 stellar templates from the Indo-U.S. library (Valdes et al. 2004). This subset was chosen to cover in a uniform manner stellar parameters like effective temperature, surface gravity, and metallicity. The choice of stellar templates, rather than SSP templates, results in a slightly better fit to the $\mathrm{H} \beta$ absorption feature and serves as a check that our choice of stellar templates is not biasing our results. To derive the mean stellar velocity and velocity dispersion we again use the pPXF method (Cappellari \& Emsellem 2004), and derive errors via Monte Carlo simulations. More details about these steps to extract stellar kinematics are given in J. Falcon-Barroso et al. (in preparation). We observe very different stellar kinematics compared to the ionized gas, which we discuss in more detail in Section 3.1.

\section{RESULTS}

While $\mathrm{H} \alpha$ is a well established tracer of the ionized gas in galaxies, with the far-IR fine-structure lines we have the opportunity to trace the ionized gas through the [O III] $88 \mu \mathrm{m}$ and $\left[\mathrm{N}_{\mathrm{II}}\right] 122 \mu \mathrm{m}$ emission without the high dust extinction suffered in the optical. Further, the [O I] $63 \mu \mathrm{m}$ line allows us to trace the neutral atomic gas, and [C II] $158 \mu \mathrm{m}$ traces both the neutral atomic gas and the ionized medium.

In Figure 2 we see that all far-IR line emission is strongly peaked toward the galaxy center, with the extent very well matched to the optical disk. The center of the line emission agrees within the astrometric uncertainties to the center seen in the stellar mass as traced by the $3.6 \mu \mathrm{m}$ emission (Figure 1) and with the kinematic center determined from the $\mathrm{CO}$ velocity field (Tsai et al. 2009). We see clearly the benefit of long wavelength observations, as the center imaged in the optical is obscured by a strong dust lane (Figures 1 and 3).

In galactic winds, neutral and ionized gas emission originates mainly from within the walls of the outflow cone (Veilleux et al. 2005). This material is expected to be turbulent and shocked due to interactions with the hot wind, resulting in increased excitation in the gas. Emission from opposite sides of the cone can produce line splitting, when seeing the opposing walls together in projection, however, in NGC 2146 we expect the geometry will make this difficult to observe. The parts of the outflow cone we see are essentially perpendicular to the line of sight, with the opposing walls of the cone well separated in projection. Broadened emission lines provide an additional outflow signature, and can also arise from turbulence in the emitting material within the cone walls. In NGC 2146, outflow signatures are observed both in the line kinematics and the gas excitation as we discuss below.

\subsection{Kinematic Signatures}

\subsubsection{Velocity Dispersion}

In NGC 2146, an increased velocity dispersion is observed in all of the far-IR emission lines (Figure 2) both along the minor axis and in regions offset above and below the disk. The different lines show some variation in the line dispersion, broadly consistent with what is expected as they originate from different gas phases. The [N II] emission displays the largest velocity dispersion, consistent with shock excitation affecting a large volume of low ionized gas. The [C II] emission arises from both the ionized and neutral phases, and the overall agreement with the neutral gas traced by [OI] suggests that the neutral phase may be dominating the emission mechanism. As [O III] arises from higher ionization material, this could account for the lower dispersion observed. All four lines show very similar morphology, providing a consistent picture of the outflow.

For the remainder of our discussion, we focus on only [C II] and $\left[\mathrm{O}_{\mathrm{I}}\right]$, as $[\mathrm{C} \mathrm{II}]$ has the highest signal-to-noise ratio but [O I] has better instrumental velocity resolution. We observe a deconvolved dispersion of $250 \mathrm{~km} \mathrm{~s}^{-1}$ in the [C II] and [O I] lines, which reflects an increase of $50-100 \mathrm{~km} \mathrm{~s}^{-1}$ above the velocity dispersion measured in the disk. Multiple components may be present but are not resolved at the $\sim 100 \mathrm{~km} \mathrm{~s}^{-1}$ instrumental resolution, and all line emission along the minor axis is centered at the systemic velocity (Figure 2). This is fully consistent with the previously established picture of the outflow geometry that results in an alignment of the far side of the cone to the north and the near side of the cone to the south perpendicular to the line of sight (Greve et al. 2000; Tsai et al. 2009), with the line emission from the opposing cone walls overwhelmed in projection by the bright central disk. 


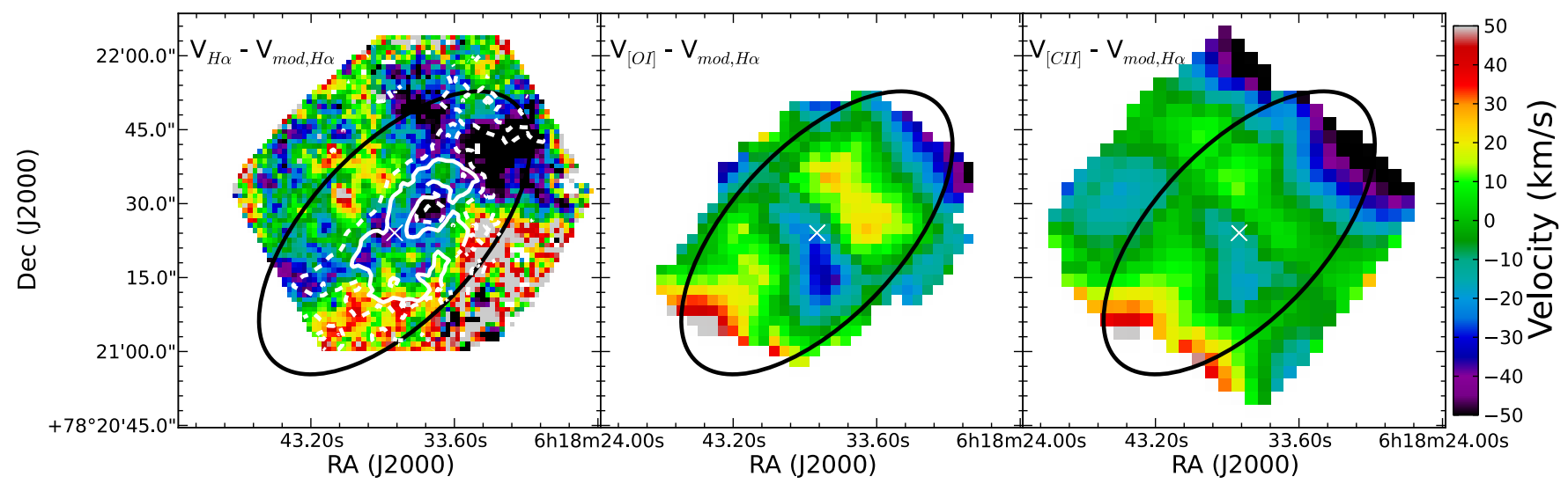

Figure 5. Residuals velocity fields in $\mathrm{H} \alpha$ (left), [O I] (center), and [C II] (right) subtracting a model velocity field based on the H $\alpha$ rotation curve (Greve et al. 2006). Regions in the $\mathrm{H} \alpha$ map along the disk major axis that show blueshifted components compared to disk rotation anti-correlate with regions of high extinction as judged from the Balmer decrement (outlined in white at $A_{\mathrm{V}}=3 \mathrm{mag}$ (dashed) and $A_{\mathrm{V}}=4 \mathrm{mag}$ (solid)), suggesting these correspond to $\mathrm{H}$ II regions foreground to the dust lane that runs along the major axis (also visible in Figure 3). Along the major axis of the PACS maps we see irregularities in the kinematics at the disk center. Above the disk there is a patch of blueshifted emission and below an extended region of redshifted emission as seen in the $\mathrm{H} \alpha$ velocity field residuals. There is no corresponding shifted emission in the PACS line maps.

(A color version of this figure is available in the online journal.)

This increase is well in excess of the increased velocity dispersion that results from beam smearing due to low spatial resolution observations of an edge-on disk (e.g., Tamburro et al. 2009; Epinat et al. 2010). We model this effect in our far-IR observations by constructing a two dimensional exponential disk model with $63^{\circ}$ inclination, to which we assign a velocity at each position based on the $\mathrm{H} \alpha$ rotation curve model of Greve et al. (2006). From this we construct a data cube with flux in a single channel (corresponding to an intrinsic $\sigma=0 \mathrm{~km} \mathrm{~s}^{-1}$ ), smooth this to the $\sim 0.8 \mathrm{kpc}$ resolution of our observations, then fit a Gaussian profile to each position in the disk. The resulting increase in central velocity dispersion reaches $\sim 80 \mathrm{~km} \mathrm{~s}^{-1}$ over a narrow $0.5 \mathrm{kpc}$ region along the major axis and a more extended $2.5 \mathrm{kpc}$ region along the minor axis. This is substantial, but significantly less than the $\sim 250 \mathrm{~km} \mathrm{~s}^{-1}$ velocity dispersion that we measure.

Tsai et al. (2009) also found evidence in the CO kinematics for a nuclear disk with a higher rotation velocity extending $300 \mathrm{pc}\left(4^{\prime \prime}\right)$ in radius. Such a nuclear disk would be unresolved in our observations, but could potentially contribute to beam smearing in the center. However, given the uniformity in the increased dispersion both along the minor axis as well as out in the wind region, we believe that this indicates that we truly are tracing the wind back to the central wind-launching region. This is very similar to what is seen in M82 (Contursi et al. 2013). In NGC 2146 the region of increased dispersion is not resolved in [O I], but the area with dispersions above $200 \mathrm{~km} \mathrm{~s}^{-1}$ extends less than $1 \mathrm{kpc}$ at the disk center and is slightly offset to the north of the disk kinematic center but agrees within the PACS astrometric uncertainty. The outflow is cylindrical out to approximately $500 \mathrm{pc}$, and broadens beyond this point into conical outflows. The outflow extends to the edge of the field of view, approximately $2.5 \mathrm{kpc}$ above the disk, and presumably even further above the disk. The region northeast of the disk shows an opening angle of $\sim 90^{\circ}$, to the southwest the opening angle is much broader, approximately $120^{\circ}$.

Although our optical spectral resolution is comparable to that of [C II], we do not observe large $250 \mathrm{~km} \mathrm{~s}^{-1}$ velocity dispersions in $\mathrm{H} \alpha$. However, due to the overall lower signal-tonoise ratio of the optical emission lines, we also cannot reliably decompose velocity dispersions in the optical below $250 \mathrm{~km} \mathrm{~s}^{-1}$ from the instrumental velocity dispersion. We do see increased velocity dispersion in the [N II] lines (at least a factor of two or more above the disk value up to about $250 \mathrm{~km} \mathrm{~s}^{-1}$ ) to the southwest in the area of the outflow. The outflow can also be considered in light of higher velocity resolution Fabry-Perot measurements of the $\mathrm{H} \alpha$ line emission in this galaxy as part of the GHASP survey (Epinat et al. 2008). These data clearly show multiple components, a detailed breakdown of which is beyond the scope of this paper but has been investigated in detail using long slit spectra (Armus et al. 1995; Greve et al. 2000). This supports the idea that a complex structure exists in the emitting material.

\subsubsection{Residual Velocity Field}

To search for further kinematic evidence of outflows we have constructed a two-dimensional model velocity field from the rotation curve measured by Greve et al. (2006) from longslit $\mathrm{H} \alpha$ kinematics. We considered also the rotation curve measured by Tsai et al. (2009) based on the CO velocity field, however, it does not extend far enough radially to include the flattened part of the rotation curve and provides a nearly identical picture of the non-circular motions. The $\mathrm{H} \alpha$ rotation curve extends to larger radii, although extinction may bias the determination of the line-of-sight velocity centroid. We subtract this model velocity field from our Balmer line, [O I] and [C II] velocity fields and consider the residual velocity fields (Figure 5).

The residual velocity fields show extended blueshifted regions along the major axis that correspond to the position of bright H II regions foreground to the dust lane (Figure 5, left), as seen in the $\mathrm{H} \alpha$ line maps. Similar to results presented in Armus et al. (1995), the residual velocity field also shows blueshifted emission to the northeast of the disk and patchy redshifted emission to the southwest that are separated by about $100 \mathrm{~km} \mathrm{~s}^{-1}$. This emission was not detected by Greve et al. (2000), however, it is clear in our maps that this detection would be extremely dependent on the exact slit position. The redshifted region corresponds approximately to the southern shock-excited region (see Section 3.2 and Figure 6). The extent of this region agrees well with the position of the anomalous CO clumps identified by Tsai et al. (2009), however they find much lower redshift velocities of $\sim 10 \mathrm{~km} \mathrm{~s}^{-1}$. The blueshifted region is 

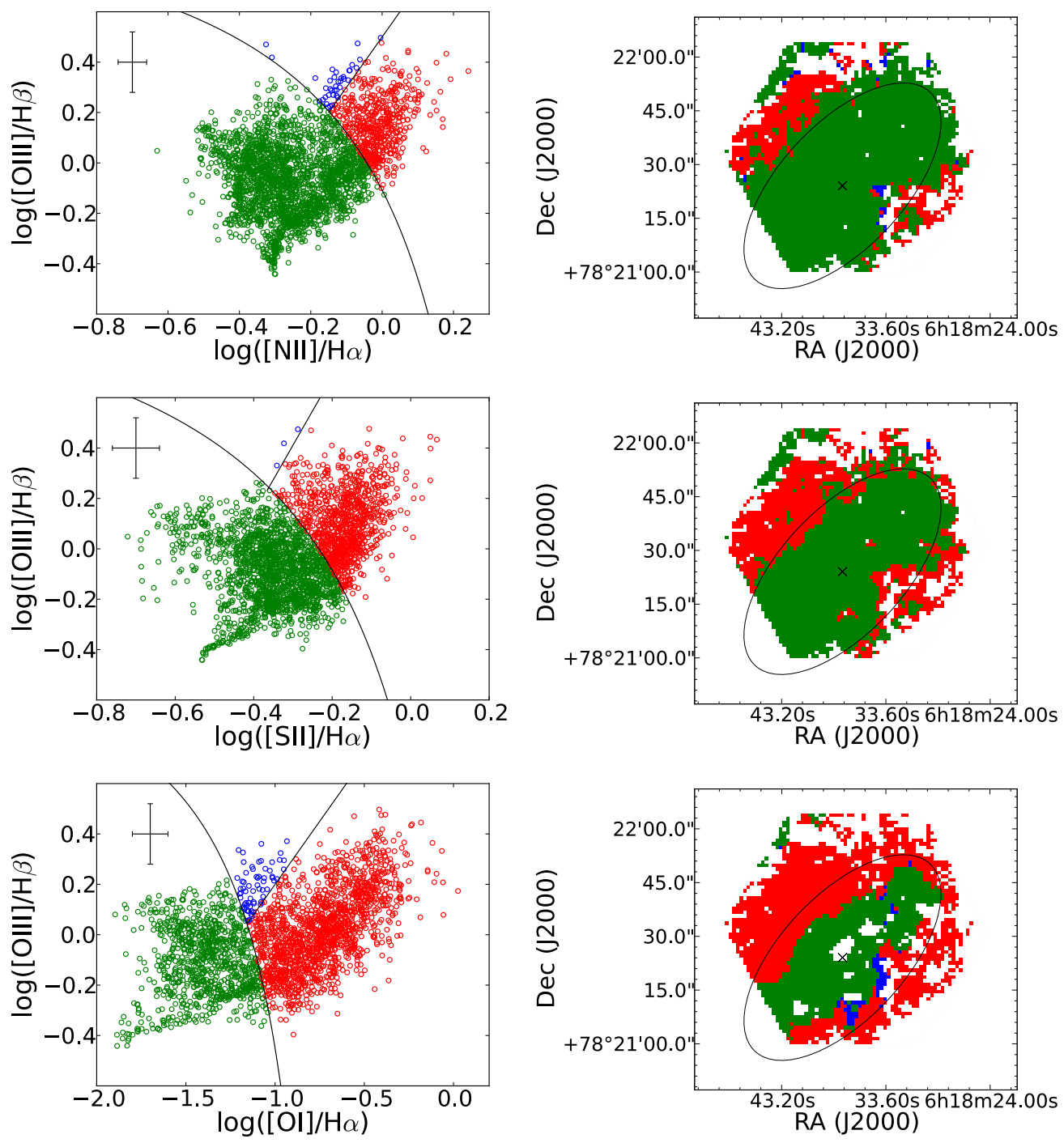

Figure 6. Left: spatially resolved BPT diagrams for three optical diagnostic tracers ([N II]/H $\alpha$, [S II]/H $\alpha,[\mathrm{O}$ I]/H $\alpha$ ). We see enhanced [O I], [S II], and [N II]/H $\alpha$ line flux ratios, beyond what is expected given an extreme starburst limit (curved line; Kewley et al. 2001) and excluded by fiducial AGN indicators (straight line; Sharp \& Bland-Hawthorn 2010). Right: corresponding maps of the color-coded regions, as identified in the figures at left. The stellar disk and kinematic center (black) is marked for reference.

(A color version of this figure is available in the online journal.)

much more compact than the shocked region, and aligns with the western edge of the molecular superbubble identified by Tsai et al. (2009) (see also Section 4.1). Both the redshifted and blueshifted regions appear to originate offset from the disk minor axis.

The PACS line residuals are large along the disk major axis (Figure 5, right). This may be evidence that our model is missing a nuclear disk component, previously observed in the CO kinematics (Tsai et al. 2009), however with a 300 pc radius that disk should be unresolved in both [O I] and [C II]. Another cause could be that we have neglected any radial motions in the disk, which may be expected since this starburst is presumably fed through significant gas inflow. However, the residuals from modeling the CO disk by Tsai et al. (2009) show no such patterns. Neither [C II] nor [O I] show evidence for significant redshifted or blueshifted material, particularly in the regions where this is seen in the $\mathrm{H} \alpha$ kinematics. It is possible the $\mathrm{H} \alpha$ emission comes from clumpy features associated with the foreground and background spiral arms, while the far-IR lines, unbiased by extinction, mainly trace gas in the main disk and outflow.

\subsection{Diagnostic Line Ratios}

Optical line ratios can be used to establish the ionization and excitation state of gas in galaxies (Baldwin et al. 1981; Kauffmann et al. 2003), though they have most commonly been applied to either integrated galaxy spectra or nuclear spectra when trying to distinguish nuclear excitation sources (SF, LINER, AGN). However, similar physics applies in resolved regions within a galaxy, where the excitation sensitive indicators $[\mathrm{N}$ II $] / \mathrm{H} \alpha,\left[\mathrm{S}_{\mathrm{II}}\right] / \mathrm{H} \alpha$, and $\left[\mathrm{O}_{\mathrm{I}}\right] / \mathrm{H} \alpha$ should be enhanced in regions of the outflow, as expected from shocked gas. Due to the high signal to noise the $[\mathrm{N} \mathrm{II}] / \mathrm{H} \alpha$ map is the most complete, although due to the high inclination and significant attenuation we still may not probe that far into the disk along the major axis. The density diagnostic $[\mathrm{S}$ II $] \lambda 6717 /[\mathrm{S}$ II $] \lambda 6731$ assuming a gas temperature of $10,000 \mathrm{~K}$ shows a fairly uniform density of $n_{e} \sim 100 \mathrm{~cm}^{-3}$.

Evidence for shock excitation along the minor axis of NGC 2146 has been previously identified through optical excitation diagnostics (Armus et al. 1995; Hutchings et al. 1990; Greve et al. 2000). Here, combining line ratio maps pixel by pixel to 


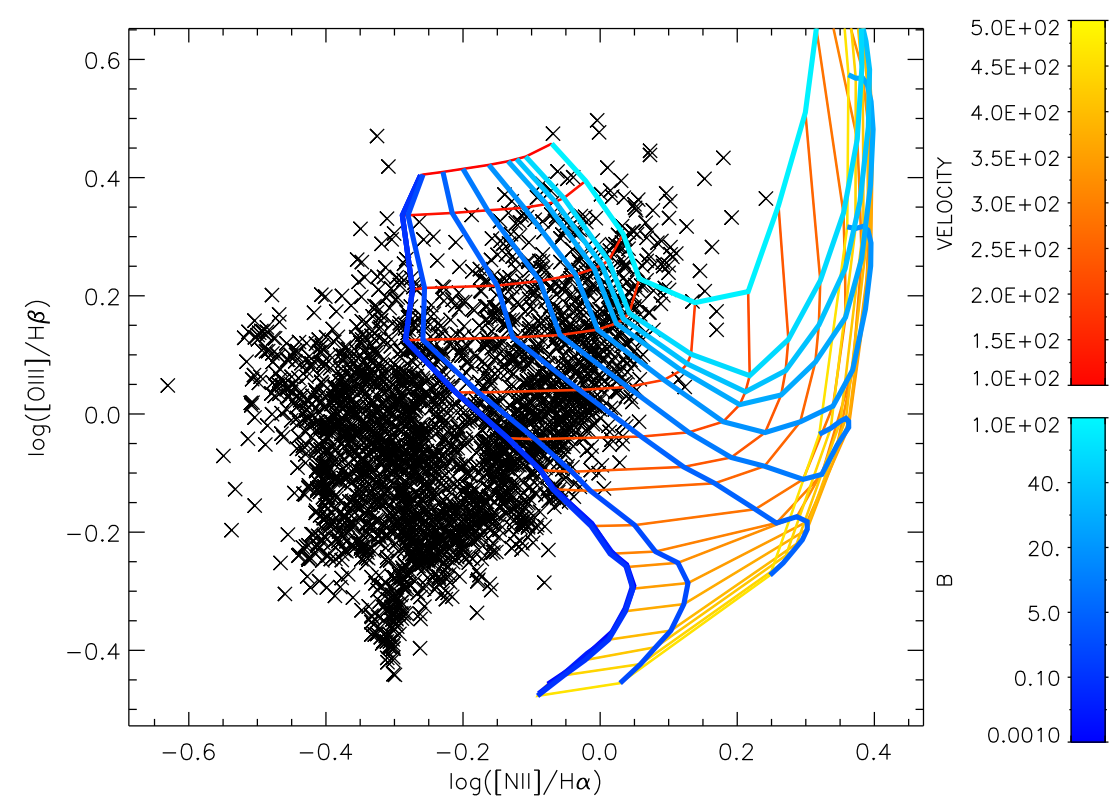

Figure 7. Shock modeling with solar metallicity models assuming $T_{e}=10,000 \mathrm{~K}$ and $n_{e}=100 \mathrm{~cm}^{-3}$ for the resolved BPT diagram shown in Figure 6. Model grid parameters are the shock velocity and B, the transverse component of the preshock magnetic field (Allen et al. 2008). Modeled shock velocities within NGC 2146 range from $100-200 \mathrm{~km} \mathrm{~s}^{-1}$, though we note that the contribution from photoionized regions that fall along the line of sight will bias us toward lower shock velocities.

(A color version of this figure is available in the online journal.)

construct BPT diagnostic diagrams (Baldwin et al. 1981), we see increased flux in the low excitation lines ([N II], [O I], [S II]) compared to $\mathrm{H} \alpha$ throughout this central region (Figure 6, left). These ratios are selected to involve lines nearby in wavelength, such that reddening due to dust extinction will not be significant and can be neglected. In all three tracers, we see evidence for high excitation (in red) beyond what is expected given an extreme starburst limit (curved line; Kewley et al. 2001) and excluded by fiducial AGN indicators (straight line, Sharp \& Bland-Hawthorn 2010). By restricting ourselves to only high confidence emission line detections we have ruled out any bias due to stellar absorption corrections or other systematic errors in these lower surface brightness regions.

Mapping these regions to their corresponding physical locations within the galaxy (Figure 6, right), the high excitation regions appear located $\sim 2 \mathrm{kpc}$ above and below the galaxy disk, and extend $\sim 5 \mathrm{kpc}$ horizontally above the disk all the way to the edge of the field of view, and is consistent with shock excitation. A combination of star forming and shocked regions along the line of sight would likely mask any shocked emission closer to the disk. Shock excitation of this sort above the disk has often been seen in longslit studies of starburstdriven galactic superwinds (Armus et al. 1989; Heckman et al. 1990), and our map appears very similar to the starburst-driven winds studied by Sharp \& Bland-Hawthorn (2010) in their study of 10 galaxies with galactic winds using IFS observations. No high ionization nuclear source is seen in the [O III]/ $\mathrm{H} \beta$ data, which is unsurprising as the nuclear region is highly attenuated.

The high excitation is fully consistent with the effects of shocks, though we cannot rule out a contribution from other low level ionization sources. We model this region using ITERA (Groves \& Allen 2010) and the MAPPINGS III library of fast radiative shocks (Allen et al. 2008), and find it is well reproduced by relatively slow shock velocities between $100-200 \mathrm{~km} \mathrm{~s}^{-1}$ (Figure 7). This modeled shock velocity is consistent between all three BPT diagrams, and agrees with previous examination of the shocks in this system (Armus et al. 1995). Modeling is complicated by the combination of stellar photoionization within the disk with shocked region above the disk due to projections along the line of sight. Given that the stellar photoionization contamination will move values down and to the left on the BPT diagram, thus resulting in lowered model shock velocities, we expect that the highest velocities measured $\sim 200 \mathrm{~km} \mathrm{~s}^{-1}$ are most representative of the shock velocities throughout the superwind.

Far-IR fine-structure line ratios provide diagnostics for the physical conditions within photodissociation regions (e.g., Kaufman et al. 1999). We map the diagnostic far-IR line ratios [O I] /[C II], [O III]/[C II], [O III]/[O I ] and [O III]/[N II] and, using the PACS $160 \mu \mathrm{m}$ image as a proxy for the continuum, the $\left[\mathrm{O}_{\mathrm{I}}\right] / 160 \mu \mathrm{m}$ and $\left[\mathrm{O}_{\mathrm{III}}\right] / 160 \mu \mathrm{m}$ ratios (Figure 8). Due to significant variations in the beam size with wavelength, PACS line maps for each ratio are convolved to the lower resolution map in the pair using kernels from Aniano et al. (2011). The more excitation sensitive indicators, $\left[\mathrm{O}_{\mathrm{III}}\right] /[\mathrm{C} \mathrm{II}]$ and $[\mathrm{O} \mathrm{III}] /$ $[\mathrm{O} I]$, show similar features, emphasizing the star formation dominated regions along the disk major axis. The $[\mathrm{O}$ III $] /[\mathrm{C}$ II $]$ ratio shows a value consistent with that found in the starburst ring of NGC 1097 (Beirão et al. 2010) and with normal star forming galaxies (Malhotra et al. 2001). The morphology in the $[\mathrm{OI}] /[\mathrm{CII}]$ map is different from emission arising from the disk. It follows the wind, suggesting that the $\left[\mathrm{O}_{\mathrm{I}}\right]$ may be enhanced in the wind, similar to the enhancement in the optical [OI] seen in the shocks. This is further seen as [O I] $/ 160 \mu \mathrm{m}$ shows an increase that aligns with the outflow region above the disk. [O III] $/ 160 \mu \mathrm{m}$ is uniform except for an increase along the major axis at the field edge. [OI]/[C II] shows much lower values (0.2-0.4) compared to M82 (Contursi et al. 2013 ; see also Section 4.2), but is consistent with the value found by Appleton et al. (2013) in the shock-heated diffuse gas filament within Stephen's Quintet. 

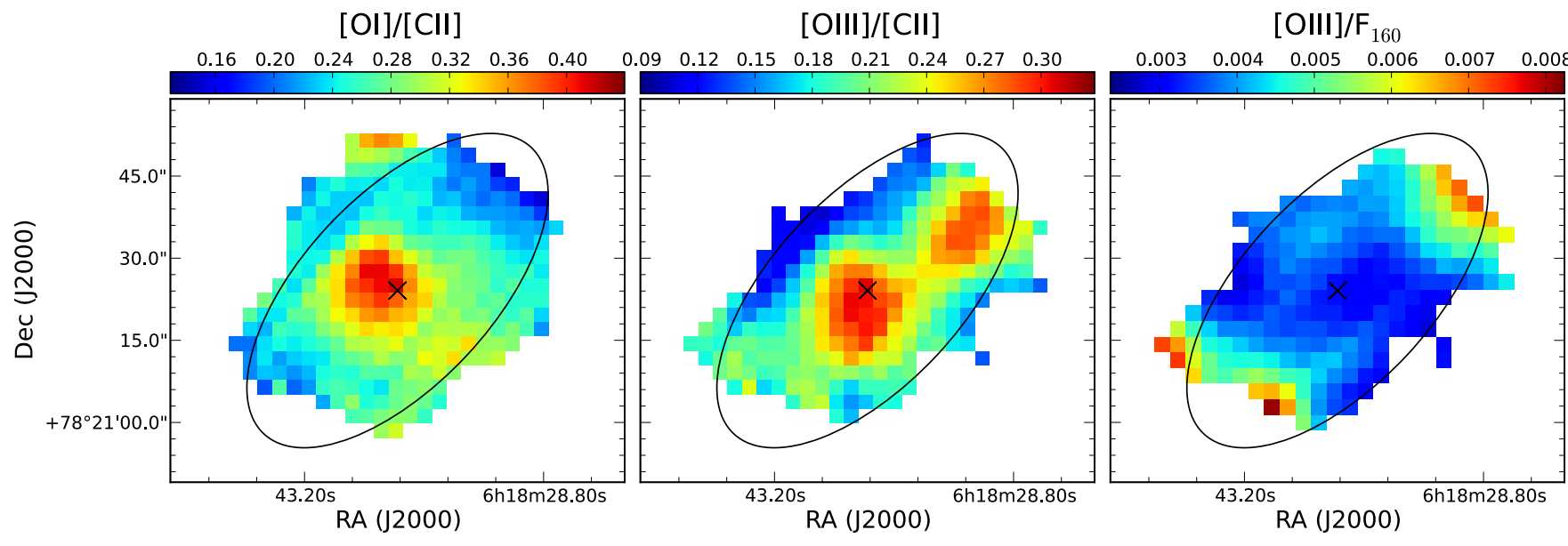

$[\mathrm{OIII}] /[\mathrm{OI}]$
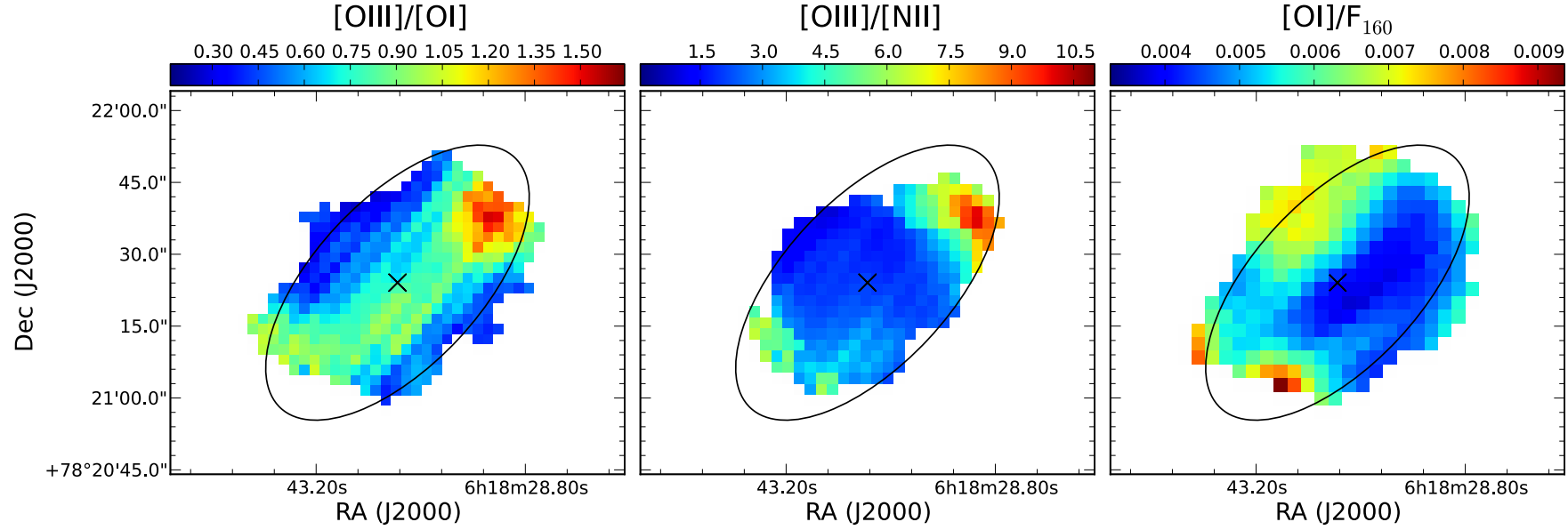

Figure 8. Line ratios of the far-IR fine-structure lines. The $\left[\mathrm{O}_{\mathrm{III}}\right] /[\mathrm{C} \mathrm{II}]$ and $[\mathrm{O} \mathrm{III}] /[\mathrm{O} \mathrm{I}]$ ratios are consistent with star formation within the disk. The $[\mathrm{O} \mathrm{I}] /[\mathrm{C}$ II $]$ ratio shows a different morphology, with a bright nucleus and a structure of high $[\mathrm{O} \mathrm{I}] /[\mathrm{C}$ II $]$ that lies perpendicular to the disk, tracing the outflow. Uncertainties are typically $5 \%$. The stellar disk and kinematic center (black) are marked for reference.

(A color version of this figure is available in the online journal.)

\subsection{Mass Entrainment in the Outflow}

In M82 substantial amounts of dust and gas have been detected in the outflow. We consider here the possibility of entrainment within the outflow of NGC 2146.

Assuming a Calzetti et al. (2000) attenuation law, Kreckel et al. (2013) converted the observed reddening in the Balmer decrement into a map of the $V$-band attenuation, $A_{\mathrm{V}}$, across NGC 2146. The Balmer line emission is able to probe down to $A_{\mathrm{V}}$ of 4-5 mag, corresponding to only a few percent of the Balmer line flux, potentially only probing the surface of the dust layer and opaque to background emission. However, given these limitations it appears that most of the dust lies in a relatively thin disk along the major axis but does exhibit a clumpy morphology (see also Section 4.1).

We trace the dust distribution through the far-IR continuum emission, detecting warm dust in the Spitzer MIPS $24 \mu \mathrm{m}$ and SOFIA FORCAST $37 \mu \mathrm{m}$ bands and the cold dust in the Herschel PACS $100 \mu \mathrm{m}, 160 \mu \mathrm{m}$ and SPIRE $250 \mu \mathrm{m}$ bands (Figure 9). The emission is expected to be dominated by thermal emission from dust, and displays a disk-like morphology. The extent in the warm dust at $24 \mu \mathrm{m}$ is well matched to the star formation as seen in the $\mathrm{H} \alpha$ emission (Figure 3), including emission from outlying $\mathrm{H}$ II regions in the north. The small amount of extended emission above and below the disk is a factor of five lower surface brightness than similar regions in M82
(Engelbracht et al. 2006), and unlike in M82 it displays a smooth morphology. This region is also significantly contaminated by diffraction spikes due to the bright central source, and we do not consider this a reliable detection of entrained material. The high resolution $37 \mu \mathrm{m}$ emission traces the extent of the central starburst, with the region of peak emission unresolved at our 3".5 resolution. This is consistent with the unresolved central extent seen in the increased [OI] $63 \mu \mathrm{m}$ velocity dispersion. In the cold dust we suffer from significantly lower angular resolution, however we generally see good agreement with the optical extent, with outlying features that generally align with the spiral arms. Note that the hexagonal pattern at low flux limits in the 100 and $160 \mu \mathrm{m}$ images is due to the shape of the PACS point-spread function seen from the bright unresolved nuclear IR emission. Careful modeling and subtraction of the disk emission would be necessary to distinguish emission due to dust in the wind (e.g., Roussel et al. 2010). However from the morphology and lack of filamentary features we conclude that these observations present no strong evidence of substantial dust entrainment at the size scales probed.

As a massive molecular outflow component has been previously identified in the superwind (Tsai et al. 2009), we estimate the atomic gas outflow mass in the superwind using the [C II] luminosity following Hailey-Dunsheath et al. (2010) 

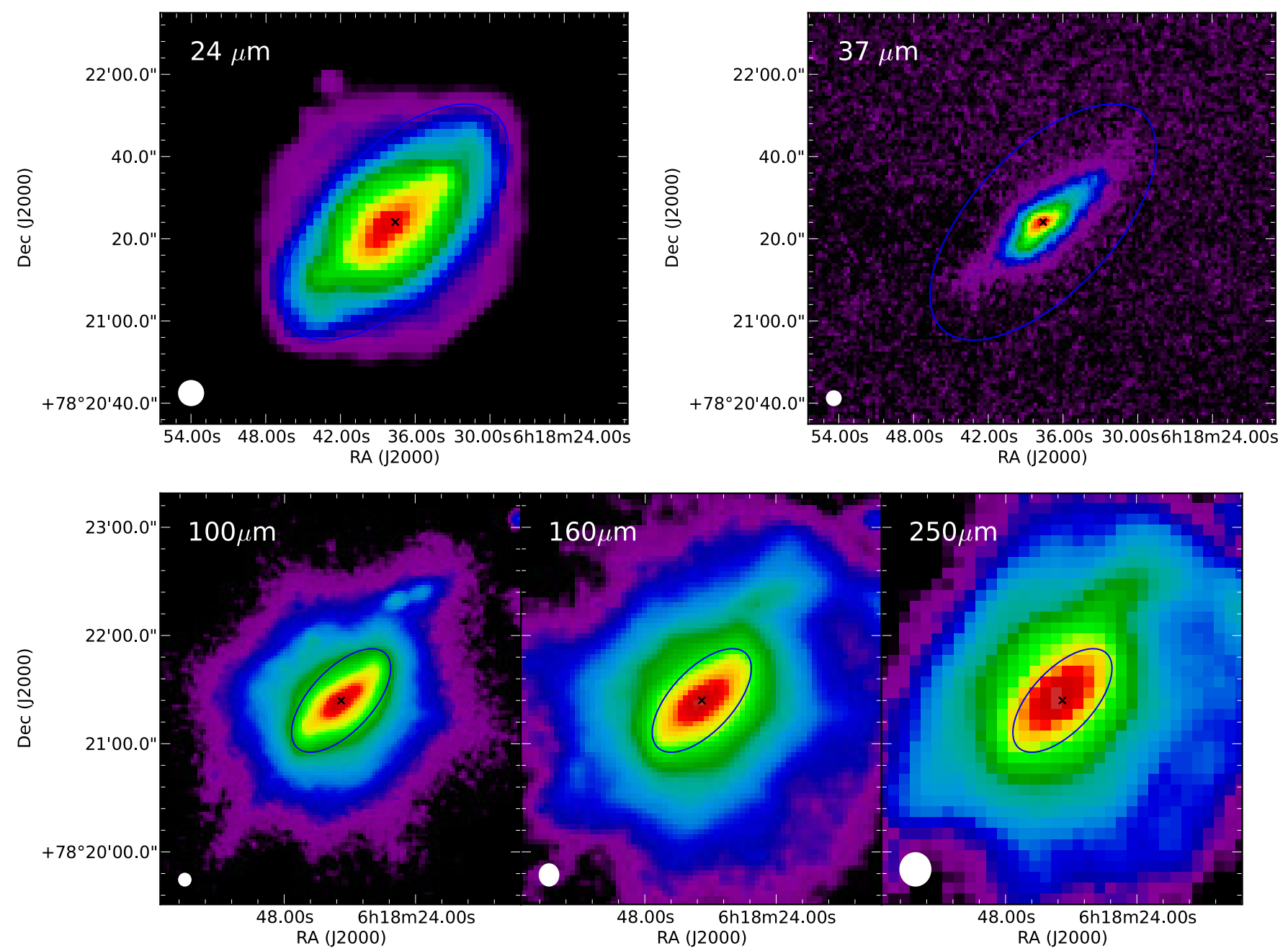

Figure 9. Dust emission in NGC 2146. Warm dust is traced by the Spitzer MIPS $24 \mu \mathrm{m}$ emission (top left) and SOFIA FORCAST $37 \mu \mathrm{m}$ emission (top right). Cold dust is traced by the Herschel PACS $100 \mu \mathrm{m}$ (bottom left), $160 \mu \mathrm{m}$ (bottom center), and SPIRE $250 \mu \mathrm{m}$ (bottom right) emission. The emission generally follows the disk of the galaxy, with extended features that trace the optical spiral arms seen extending beyond the field of view of our line maps. Hexagonal features in the PACS images at low flux levels are due to the instrumental point-spread function. The stellar disk (blue oval) and kinematic center (cross) is marked for reference and is the same in all figures.

(A color version of this figure is available in the online journal.)

(see also Maiolino et al. 2012). We estimate the mass as

$$
\begin{aligned}
\frac{M_{\text {atomic }}}{M_{\odot}}= & 0.77\left(\frac{0.7 L_{[\mathrm{C} \text { II }]}}{L_{\odot}}\right)\left(\frac{1.4 \times 10^{-4}}{X_{C^{+}}}\right) \\
& \times \frac{1+2 e^{-91 K / T}+n_{\text {crit }} / n}{2 e^{-91 K / T}},
\end{aligned}
$$

where $X_{C^{+}}$is the $\mathrm{C}^{+}$abundance per hydrogen atom, $T$ is the gas temperature, $n$ is the gas density, and $n_{\text {crit }}$ is the critical density of the $[\mathrm{C}$ II $] 158 \mu \mathrm{m}$ transition $\left(3 \times 10^{3} \mathrm{~cm}^{-3}\right)$. We assume a $\mathrm{C}^{+}$abundance typical of Milky Way photodissociation regions $\left(X_{C^{+}}=1.6 \times 10^{-4}\right.$; Sofia et al. 2004), a temperature of $200 \mathrm{~K}$, and a density much higher than the critical density $\left(n_{\text {crit }} / n \ll 1\right)$. We assume the high density case as this provides a lower limit for the total atomic gas mass. This calculation is relatively insensitive to the temperature chosen; a choice of $1000 \mathrm{~K}$ instead of $200 \mathrm{~K}$ would only result in a $15 \%$ change in the total mass. Sofia et al. (2011), using a different methodology, suggests a lower value of $X_{C^{+}}$, which would also result in a more massive wind. We emphasize that, in general, this mass estimate provides a lower limit in the case that we have a lower gas density or temperature.

If we assume that all the [C II] emission from where we identify the superwind through the increased [C II] velocity dispersion is due to a massive outflow (with no disk component), we find $L_{[\mathrm{C} \text { II }]}=3.0 \times 10^{9} L_{\odot}$ in the northeast and $L_{[\mathrm{C} \text { II }]}=1.5 \times$ $10^{9} L_{\odot}$ in the southwest to give a total $M_{\text {atomic }}>3.9 \times 10^{9} M_{\odot}$. However, given the $63^{\circ}$ inclination, we may not be cleanly separating the disk contribution from the [C II] emission in the outflow.

As we cannot kinematically isolate the contribution of [C II] emission due to the outflow from [C $\mathrm{CI}]$ in a disk component, we fit the disk intensity in regions with low velocity dispersion using a two dimensional exponential disk model, and consider the residual emission in the regions identified as dominated by the superwind through the increased [C II] velocity dispersion (Figure 10). The residuals within the disk center are clumpy, however outside they are smooth and indicate an excess of flux above the simple disk model. We note, however, that our disk modeling is sensitive to the high inclination angle of the disk, as in projection the $30^{\prime \prime}$ distance angular extent 

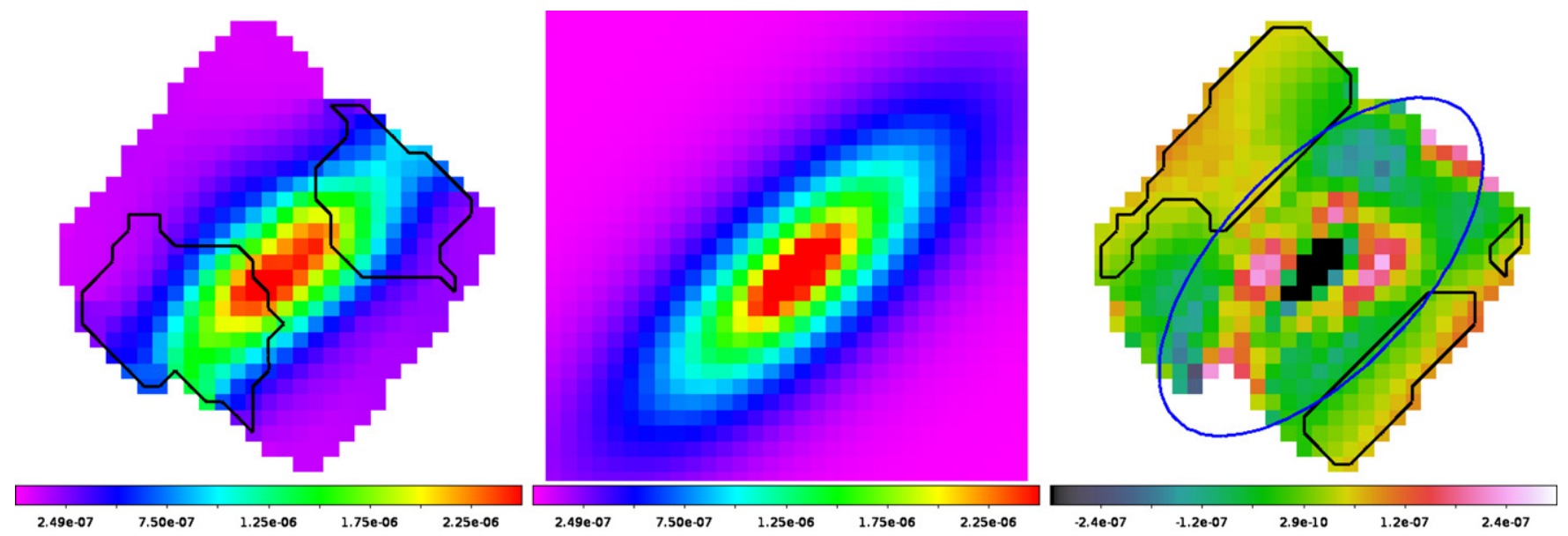

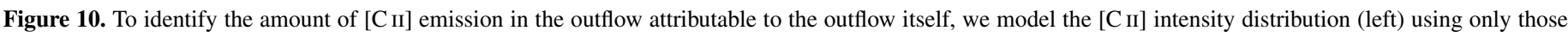

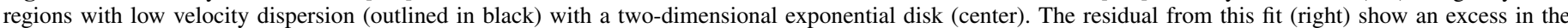

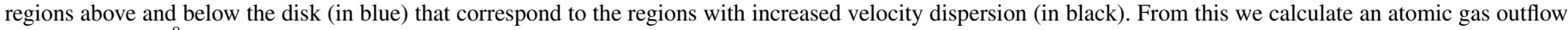

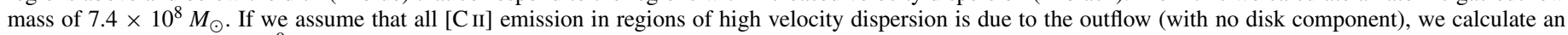
atomic gas mass of $3.9 \times 10^{9} M_{\odot}$.

(A color version of this figure is available in the online journal.)

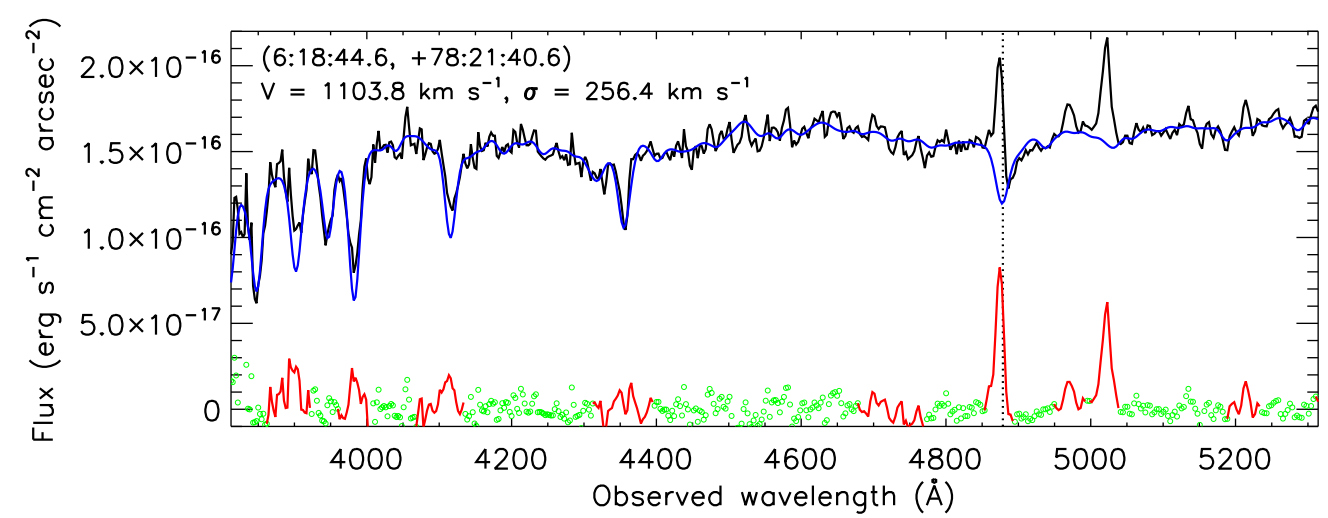

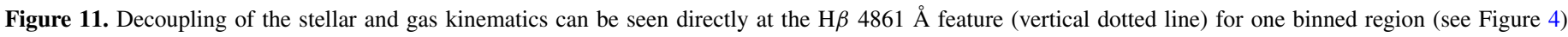

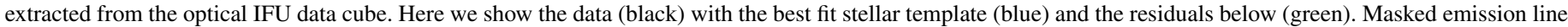

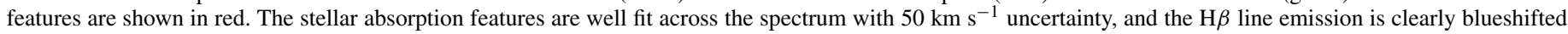
from the underlying stellar absorption feature.

(A color version of this figure is available in the online journal.)

observed along the minor axis covers a large radius $(\sim 6 \mathrm{kpc})$, and requires significant extrapolation from the central $5 \mathrm{kpc}$ diameter region we are modeling. We find that that $\sim 20 \%$ of the line flux is contributed by the outflow, which corresponds to $L_{\left[\mathrm{C}_{\text {II }}\right]}=6.8 \pm 0.8 \times 10^{8} L_{\odot}$ in the northeast and $L_{\left[\mathrm{C}_{\text {II }}\right]}=$ $1.9 \pm 0.3 \times 10^{8} L_{\odot}$ in the southwest. This gives a total atomic mass outflow of $M_{\text {atomic }}>7.4 \times 10^{8} M_{\odot}, 40 \%$ higher than what was found in the molecular superbubbles and outflows $\left(5.1 \times 10^{8} M_{\odot}\right.$; Tsai et al. 2009). This is also a substantial amount as compared to the molecular $\left(2 \times 10^{9} M_{\odot}\right.$; Young et al. $1988)$ or the atomic hydrogen $\left(1.6 \times 10^{9} M_{\odot}\right.$; Taramopoulos et al. 2001) gas masses measured in the central disk.

\subsection{Stellar Kinematics}

Our fits to the stellar kinematics show no ordered motions on $5 \mathrm{kpc}$ scales in the central stellar disk within the $\sim 50 \mathrm{~km} \mathrm{~s}^{-1}$ uncertainty, in stark contrast to the gas kinematics that show a large $\sim 500 \mathrm{~km} \mathrm{~s}^{-1}$ velocity gradient across the same region. Voronoi binning (Cappellari \& Copin 2003) of neighboring spectra to ensure a minimum signal to noise of 20 in the stellar continuum confirms that this is not a signal to noise issue (Figure 4, right). However, we cannot exclude the presence of ordered motion on larger scales, or along the disk midplane where the dust extinction is extreme. We directly observe the offset between the stellar and gas kinematics in individual binned regions by examining the $\mathrm{H} \beta 4861 \AA$ feature (Figure 11). Here, the underlying stellar absorption is clearly misaligned from the superposed nebular emission line.

This is in contrast to findings by previous studies in NGC 2146 of the stellar absorption features in longslit and driftscan spectra (Kobulnicky \& Gebhardt 2000; Greve et al. 2006). This may be due to significant dust absorption of the stellar continuum resulting in unreliable stellar kinematic measurements directly along the major axis. We find that by simulating a longslit within our IFU data we can reproduce a rotation curve similar to that seen by Kobulnicky \& Gebhardt (2000), mainly by including the redshift emission to the northwest side of the disk, that covers a similar range of velocities but centered at a higher systemic velocity. However, in the two dimensional kinematics it is apparent that this does not indicate bulk rotation in the stars, and that most of the motion appears unordered. We cannot explain the $\sim 200 \mathrm{~km} \mathrm{~s}^{-1}$ offset in systemic velocity that we observe (also apparent in the longslit measurements by Kobulnicky \& 


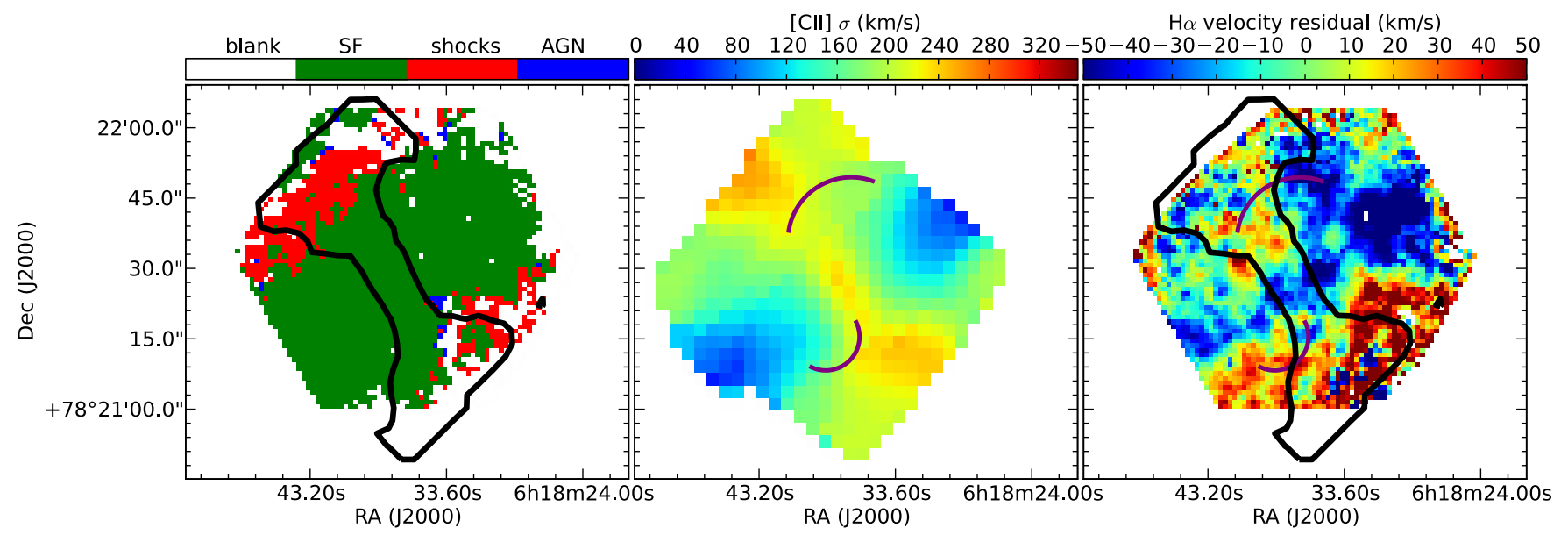

Figure 12. Comparison of different superwind indicators. Left: the optically identified shock regions (red, see Figure 6) show very good agreement with the high velocity dispersion identified in the [C II] line (black contours). Center: the location (shown here with purple contours) of the superbubble (south) and molecular outflow (north) identified by Tsai et al. (2009) appear offset from the superwind identified by the increase in [C II] velocity dispersion (in color). Right: comparing the location of the $\mathrm{H} \alpha$ velocity residual with the superbubble and outflow identified in Tsai et al. (2009) (purple contours) and with the regions of increased C II velocity dispersion (black contours) reveals no alignment.

(A color version of this figure is available in the online journal.)

Gebhardt 2000), particularly as we find it in relation to the line nebular velocities measured within our same data set. The overall agreement between the ionized gas kinematics observed in the far-IR, which should suffer very little dust attenuation, and in the optical (see Section 3.1.2 and Figure 5) suggests that any bias due to dust must be mainly affecting the stellar kinematics.

\section{DISCUSSION}

\subsection{Comparing Superwind Indicators}

The case for a superwind in NGC 2146 was first made by Armus et al. (1995), who identified soft X-ray emission consistent with swept up gas that has been shock heated. They also measured optical line diagnostics along the minor axis consistent with shock excitation and calculated that the energy from the starburst was enough to power the observed wind. This soft X-ray emission has been further resolved (della Ceca et al. 1999; Inui et al. 2005), and found to align with the CO bubbles identified by Tsai et al. (2009). Given our spatially resolved superwind indicators, we can compare these regions with the features we observe. A clear diagram of the system geometry can be found in Figure 11 of Tsai et al. (2009).

The regions consistent with shock contributions to the gas excitation (Figure 6) align with the diffuse soft X-ray emission mapped by Chandra (Inui et al. 2005). These regions are also relatively well aligned with the regions of increased velocity dispersion seen in the PACS line maps (Figure 12, left). This is fully consistent with a conical outflow both above and below the disk.

The shocked region extends beyond the boundaries of the CO bubbles identified by Tsai et al. (2009), and the bubbles appear misaligned with the regions of increased [C II] velocity dispersion (Figure 12, center). Given the three dimensional nature of this outflow, the shock emission may lie along different filamentary channels than the molecular gas.

The features in the $\mathrm{H} \alpha$ residual velocity field seen above and below the disk after subtracting the Greve et al. (2006) model rotation curve are very difficult to understand and do not align perfectly with the outflow regions identified, as we would expect if they originated from the cone walls (Figure 12, right). The blueshifted region north of the disk falls mostly to the west of the outflow, and exists over a very narrow patch of that region. The redshifted emission to the southwest does overlap with the outflow, but is also offset to the west. Perhaps these residual features are simply due to irregular motions within the clearly disturbed gas disk. In addition, $\mathrm{H} \alpha$ is quite susceptible to extinction, which will also influence the residuals measured. We see no clear residuals in the PACS lines that would correspond to blueshifted or redshifted emission along the minor axis, however this is consistent with the expected geometry of the system (see Section 3.1.1).

\subsection{Comparison with M82}

We find that NGC 2146 exhibits many features similar to the prototype starburst galaxy M82. Both benefit from a nearly edge-on viewing angle. M82 also shows similar features through the soft X-ray emission tracing the starburst-driven wind (Watson et al. 1984) and mass loaded outflows in the molecular gas (Walter et al. 2002). Both systems display multiple line components in the ionized gas, extensively studied in M82 (McKeith et al. 1995; Westmoquette et al. 2009). Though in NGC 2146 they are not resolved in our $\mathrm{H} \alpha$ data, they are apparent in the GHASP H $\alpha$ data cube (Epinat et al. 2008).

In a comparison of the dimensions of the starburst and outflow between the two systems by Greve et al. (2000), both display a similar geometry with conical outflows launched from a distributed region on the disk midplane and opening out above and below the disk. NGC 2146 is a more massive galaxy, with a stellar mass of $2 \times 10^{10} M_{\odot}$ (Skibba et al. 2011) compared to $6 \times 10^{9} M_{\odot}$ for M82 (Karachentsev et al. 2004). Correspondingly, the outflow in NGC 2146 displays a significantly larger geometric scale in the dimensions of both the starburst and outflow regions (Greve et al. 2000), which serves to dilute the strength of the outflow through the larger starburst volume. Our observations support this idea, as we measure in NGC 2146 a large opening angle of $90^{\circ}$ and $120^{\circ}$, compared to $50^{\circ}$ for M82 (Walter et al. 2002; Contursi et al. 2013), and find no high velocity outflows in the $\mathrm{H} \alpha$ residual velocity field for the inner $\sim 5 \mathrm{kpc}$ region mapped. 
The gas excitation in M82 is dominated by stellar ionization close to the disk with an increasing contribution from shocks further away (Heckman et al. 1990; Shopbell \& Bland-Hawthorn 1998). We see this also very clearly in NGC 2146 (Figures 6 and 7), as beyond $1 \mathrm{kpc}$ above the disk the excitation is no longer consistent with photoionization and suggests a significant contribution from shocks.

The far-IR fine-structure lines in M82 were observed by Contursi et al. (2013). In NGC 2146 we see that the [C II] and [O I] show quite clearly a $\sim 100 \mathrm{~km} \mathrm{~s}^{-1}$ increase in the velocity dispersion in the superwind and along the minor axis to the disk center, very similar to what is seen in M82 and suggestive of moderate superwind velocities in the atomic gas. Both galaxies also show a relatively wide opening angle in the [C II] and [O I] diagnostics. In M82 this is different from what is seen in the $\mathrm{H} \alpha$ outflow, which is much more collimated. This is difficult to compare with NGC 2146, where we do not see the outflow clearly in $\mathrm{H} \alpha$ in our observations of the central region.

In M82 dust is seen up to $6 \mathrm{kpc}$ along the minor axis both in mid-IR continuum and polycyclic aromatic hydrocarbon emission (Engelbracht et al. 2006), although the physical properties modeled from the far-IR results suggest that much of it has been stripped by tidal interaction and not through the starburst wind (Roussel et al. 2010). The $24 \mu \mathrm{m}$ emission in the wind of NGC 2146 is at least a factor of five lower surface brightness than M82 with a smoother morphology, providing no clear evidence for significant dust entrainment (Figure 9).

\subsection{Application to High Redshift Studies}

NGC 2146 is undergoing a substantial starburst, which places it as an outlier in specific SFR(SFR $/ M_{*}=4 \times 10^{-10}$ year $^{-1}$ ) as a function of stellar mass when compared to local galaxies (Brinchmann et al. 2004). However, it is consistent with the increased specific SFR observed in high redshift $z \sim 1-3$ galaxies (Bauer et al. 2005) where outflows are commonly identified in starburst galaxies (Pettini et al. 2001; Shapley et al. 2003; Steidel et al. 2004; Weiner et al. 2009; Rubin et al. 2010). Observations of X-rays and ionized gas kinematics have proven to be strong indicators for the presence of outflows in galaxies in the local universe, however their use at high redshift is limited. The $\left[\mathrm{CII}_{\mathrm{II}}\right]$ line, a dominant coolant of the diffuse ISM that is observable with ALMA, provides a complimentary picture, tracing the morphology of the outflow through the widths of observed line profiles (see Section 3.1.1).

While for some systems an outflow may be identified from broad wings in the spectrum alone (Maiolino et al. 2012; Carilli et al. 2013), for high redshift galaxies similar to NGC 2146 spatial resolution is essential to identifying the outflow through the [C II] line emission. In the spatially integrated far-IR line profile of NGC 2146 we find that both the [O I] and the [C II] lines are well fit by two Gaussians that decompose the galaxy into two roughly equal components that represent the motion of the disk and have widths consistent with the instrumental resolution (Figure 13). We recover no broad residual component that could be attributed to an outflow. In general, outflows with a velocity equal to the circular velocity will be impossible to distinguish in unresolved data, which is especially problematic as in star formation dominated ULIRGs this is typically observed to be the case (Martin 2005). Spatially resolved observations are essential for identifying the presence of an outflow.

ALMA will be the perfect telescope for identifying superwinds through the $[\mathrm{C}$ II] line kinematics in high redshift systems, for which the galaxies M82 and NGC 2146 provide key

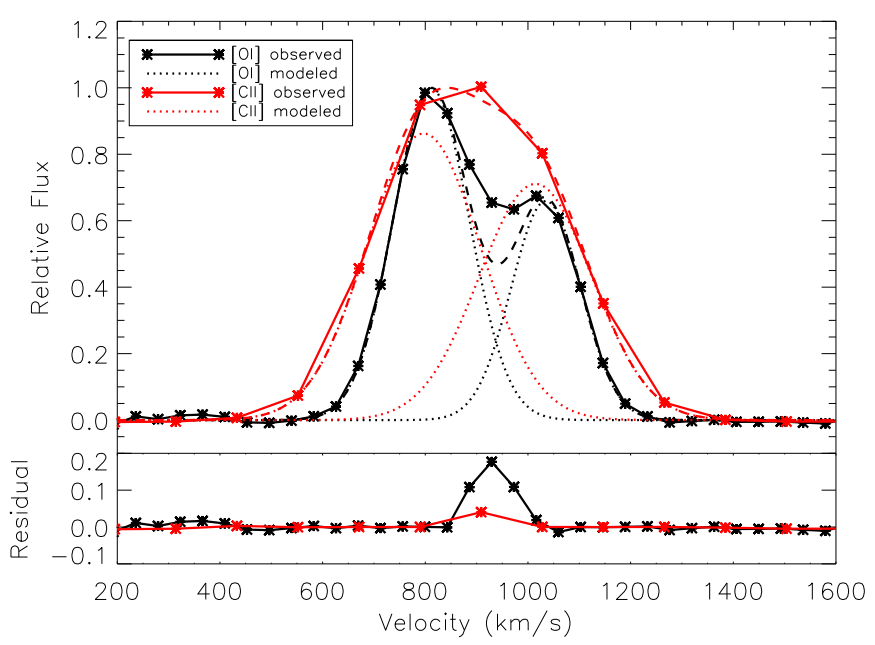

Figure 13. Modeling of spatially integrated [O $\mathrm{I}]$ and $[\mathrm{C}$ II] line profiles assuming a double Gaussian fit (top). For [C $\mathrm{II}]$ and [O I] the fits recover the instrumental resolution for the approaching and receding sides of the disk, with no broad residual (bottom) that could indicate the contribution of the outflow.

(A color version of this figure is available in the online journal.)

local analogs. The ALMA Band-9 and Band-10 receivers provide $00^{\prime \prime} 1-0^{\prime \prime} .2$ resolution for the $\left[\mathrm{C}_{\mathrm{II}}\right]$ line at $z=1-3$, with a resulting 1-2 kpc spatial resolution that is equivalent to what we obtain here for NGC 2146.

\subsection{A Transitional Galaxy}

The kinematic decoupling of the stars and gas are consistent with evidence from the optical morphology that this galaxy has undergone a recent merger event that may have disrupted the rotational support of the stars. Disjoint optical morphology and kinematics have been tied in simulations to mergers with mass ratio between 4:1 and 10:1 (Bournaud et al. 2004). This galaxy may be in the process of settling into an elliptical type galaxy, as is thought to happen with ULIRG galaxies (Kormendy \& Sanders 1992; Genzel et al. 2001).

The interaction history of NGC 2146, however, is not completely clear. There is no double-nucleus observed in the optical or IR images, implying that the nuclei may have already merged, which could explain the odd stellar kinematics in the center. Based on the extended H I tail (Taramopoulos et al. 2001), it is possible that a third galaxy also passed by the system and was tidally disrupted, creating both the extended tidal features and possibly also causing features at smaller galactocentric radius such as the non-planar $\mathrm{H} \alpha$ arc (Greve et al. 2006). In general, it is clear that NGC 2146 has not yet settled into a stable configuration, raising the question of what the final morphology of this galaxy will be. We consider two possibilities: the substantial gas reservoir could serve to stabilize and rebuild the disk, resulting in a bulge-dominated spiral galaxy, or the starburst and wind could use up and expel sufficient material to stop star formation and convert the system to an elliptical.

NGC 2146 has a substantial reservoir of gas in both the atomic and molecular phases surrounding the galaxy. Young et al. (1988) measure a total mass of $1.2 \times 10^{10} M_{\odot}$ in molecular clouds around the system, and Taramopoulos et al. (2001) detect a further $6.2 \times 10^{9} M_{\odot}$ in $\mathrm{HI}$ in an extended configuration around the galaxy. This mass in gas is roughly equal to the amount currently existing in the stars $\left(M_{*}=2 \times 10^{10} M_{\odot}\right.$; Kennicutt et al. 2011). Even considering the current high SFR of $7.9 M_{\odot} \mathrm{yr}^{-1}$ (Kennicutt et al. 2011), this galaxy has enough 
fuel to form stars for at least a further 2.3 Gyr. Presumably it will form stars for much longer, assuming a lower SFR after the starburst ends, and will rebuild a disk of significant mass.

However, this system also has a substantial wind. della Ceca et al. (1999) studied the soft X-ray emission from the starburstdriven superwind and estimated a mass loaded outflow rate of $9 M_{\odot} \mathrm{yr}^{-1}$, with energetics that suggest the hot gas may be able to escape from the galaxy. Mass loading of the outflow is also observed by Tsai et al. (2009) in the molecular outflow and two superbubbles identified from the molecular gas kinematics, and we estimate a substantial atomic gas mass of $7.4 \times 10^{8} M_{\odot}$ in the outflow as well (see Section 3.3). If we consider that the starburst only affects the central gas, measured as $2 \times 10^{9} M_{\odot}$ in molecular gas (Young et al. 1988) and $1.6 \times 10^{9} M_{\odot}$ in $\mathrm{H} \mathrm{I}_{\mathrm{I}}$ (Taramopoulos et al. 2001), then the galaxy could potentially convert or expel all of this material in only $200 \mathrm{Myr}$, a reasonable duration for a starburst (Di Matteo et al. 2008). Even without removing all of the material, if the starburst is able to create a significant halo of hot gas it may be sufficient to shock heat any of the extended gas that falls in at later times. The massive stellar spheroid may also provide the means to stabilize the gas against future star formation, through morphological quenching (Martig et al. 2009).

These two proposed scenarios are in fact extremes, with some combination of both likely responsible for the variety in the morphology and kinematics of elliptical galaxies that we see today (Emsellem et al. 2011). Detailed simulations are necessary to distinguish what evolutionary future will play out in NGC 2146, but in either case it represents a very interesting and unusual transition object.

\section{CONCLUSION}

We present Herschel PACS far-IR fine-structure line observations, optical IFU data and SOFIA $37 \mu \mathrm{m}$ observations of the starburst-driven superwind in NGC 2146, a nearby LIRG. We find high velocity dispersions in all far-IR lines, with deconvolved linewidths of $\sim 250 \mathrm{~km} \mathrm{~s}^{-1}$ that extend along the minor axis through the disk center and open into conical regions above and below the galaxy disk. This is fully consistent with the previously proposed picture of the outflow geometry that results in an alignment of the far side of the cone to the north and the near side of the cone to the south perpendicular to the line of sight (Greve et al. 2000; Tsai et al. 2009), with the line emission from the opposing cone walls overwhelmed in projection by the bright central disk. Unlike in optical studies, which are limited by the high extinction at the galaxy center, we are able to observe the superwind launching region, which extends less than one kiloparsec along the major axis in the center of the galaxy.

We present evidence for enhanced low-ionization line emission $([\mathrm{OI}] / \mathrm{H} \alpha,[\mathrm{S} \mathrm{II}] / \mathrm{H} \alpha,[\mathrm{N} \mathrm{II}] / \mathrm{H} \alpha)$ coincident with the regions of increased velocity dispersion above and below the plane seen in the far-IR emission line maps. The optical line ratios are consistent with shock excitation, though we cannot rule out a contribution from other low level ionization sources. The position of these shock and superwind indicators seen in the far-IR and optical have positions that match soft X-ray emission (Inui et al. 2005), with both the X-ray emission and CO bubbles (Tsai et al. 2009) located within the full extent of the outflow cone we identify. We do not detect dust entrainment in the outflow, in either the warm or the cold dust components. In general, the outflow geometry is very similar to that observed in M82. Outflows of this nature would not be easily discerned in unresolved [C II] observations of high redshift systems but will be resolved by ALMA at $z \sim 1-3$.

We also observe stellar kinematics that are decoupled from the gas kinematics in all phases, consistent with predictions for post-merger systems, though we may also be suffering from some bias due to dust. As the galaxy is still in the midst of its post-merger starburst it has yet to expel or transform the bulk of its molecular gas. The starburst-driven wind is potentially crucial for transformation of the galaxy's morphology into a red and dead elliptical.

Far-IR line observations provide an unobscured view of the outflow and starburst, crucial for detailed study of the physical conditions in these regions. This system will provide an important local analog for future high redshift studies of IR-luminous galaxies driving galactic-scale superwinds.

We thank the referee for helpful comments. K.K. acknowledges the support of grants GR 3948/1-1 and SCHI 536/8-1 from the DFG Priority Program 1573, "The Physics of the Interstellar Medium.” A.D.B. acknowledges support from the National Science Foundation through grant AST-0955836, as well as a Cottrell Scholar award from the Research Corporation for Science Advancement. The National Radio Astronomy Observatory is a facility of the National Science Foundation Operated under cooperative agreement by Associated Universities, Inc. F.T. acknowledges the DFG grant TA 801/1-1.

This work is based on observations made with Herschel. Herschel is an ESA space observatory with science instruments provided by European-led Principal Investigator consortia and with important participation from NASA. PACS has been developed by a consortium of institutes led by MPE (Germany) and including UVIE (Austria); KU Leuven, CSL, IMEC (Belgium); CEA, LAM (France); MPIA (Germany); INAF-IFSI/OAA/ OAP/OAT, LENS, SISSA (Italy); IAC (Spain). This development has been supported by the funding agencies BMVIT (Austria), ESA-PRODEX (Belgium), CEA/CNES (France), DLR (Germany), ASI/INAF (Italy), and CICYT/MCYT (Spain).

Based in part on observations collected at the Centro Astronómico Hispano Alemán (CAHA), operated jointly by the Max-Planck Institut für Astronomie and the Instituto de Astrofisica de Andalucia (CSIC).

Based in part on observations made with the NASA/DLR Stratospheric Observatory for Infrared Astronomy (SOFIA). SOFIA is jointly operated by the Universities Space Research Association, Inc. (USRA), under NASA contract NAS2-97001, and the Deutsches SOFIA Institut (DSI) under DLR contract 50 OK 0901 to the University of Stuttgart.

This research made use of APLpy, an open-source plotting package for Python hosted at http://aplpy.github.com.

\section{REFERENCES}

Allen, M. G., Groves, B. A., Dopita, M. A., Sutherland, R. S., \& Kewley, L. J. 2008, ApJS, 178, 20

Alonso-Herrero, A., García-Marín, M., Monreal-Ibero, A., et al. 2009, A\&A, 506,1541

Alonso-Herrero, A., García-Marín, M., Rodríguez Zaurín, J., et al. 2010, A\&A, 522, A7

Aniano, G., Draine, B. T., Gordon, K. D., \& Sandstrom, K. 2011, PASP, 123,1218

Appleton, P. N., Guillard, P., Boulanger, F., et al. 2013, ApJ, 777, 66

Armus, L., Heckman, T., \& Miley, G. 1987, AJ, 94, 831

Armus, L., Heckman, T. M., \& Miley, G. K. 1989, ApJ, 347, 727

Armus, L., Heckman, T. M., Weaver, K. A., \& Lehnert, M. D. 1995, ApJ, 445,666

Armus, L., Mazzarella, J. M., Evans, A. S., et al. 2009, PASP, 121, 559 
Arribas, S., Colina, L., Monreal-Ibero, A., et al. 2008, A\&A, 479, 687

Baldwin, J. A., Phillips, M. M., \& Terlevich, R. 1981, PASP, 93, 5

Bauer, A. E., Drory, N., Hill, G. J., \& Feulner, G. 2005, ApJL, 621, L89

Beirão, P., Armus, L., Appleton, P. N., et al. 2010, A\&A, 518, L60

Bellocchi, E., Arribas, S., Colina, L., \& Miralles-Caballero, D. 2013, A\&A, 557, A59

Bernard-Salas, J., Spoon, H. W. W., Charmandaris, V., et al. 2009, ApJS, 184,230

Bournaud, F., Combes, F., \& Jog, C. J. 2004, A\&A, 418, L27

Brinchmann, J., Charlot, S., White, S. D. M., et al. 2004, MNRAS, 351, 1151

Bruzual, G., \& Charlot, S. 2003, MNRAS, 344, 1000

Calzetti, D., Armus, L., Bohlin, R. C., et al. 2000, ApJ, 533, 682

Cappellari, M., \& Copin, Y. 2003, MNRAS, 342, 345

Cappellari, M., \& Emsellem, E. 2004, PASP, 116, 138

Carilli, C. L., Riechers, D., Walter, F., et al. 2013, ApJ, 763, 120

Contursi, A., Poglitsch, A., Grácia Carpio, J., et al. 2013, A\&A, 549, A118

Croxall, K. V., Smith, J. D., Brandl, B. R., et al. 2013, ApJ, 777, 96

de Vaucouleurs, G., de Vaucouleurs, A., Corwin, H. G., Jr., et al. 1991, Third Reference Catalogue of Bright Galaxies. Volume I: Explanations and references. Volume II: Data for galaxies between $0^{h}$ and $12^{h}$. Volume III: Data for galaxies between $12^{h}$ and $24^{h}$ (New York: Springer)

della Ceca, R., Griffiths, R. E., Heckman, T. M., Lehnert, M. D., \& Weaver, K. A. 1999, ApJ, 514, 772

Di Matteo, P., Bournaud, F., Martig, M., et al. 2008, A\&A, 492, 31

Emsellem, E., Cappellari, M., Krajnović, D., et al. 2011, MNRAS, 414, 888

Engelbracht, C. W., Kundurthy, P., Gordon, K. D., et al. 2006, ApJL, 642, L127

Epinat, B., Amram, P., Balkowski, C., \& Marcelin, M. 2010, MNRAS, 401,2113

Epinat, B., Amram, P., \& Marcelin, M. 2008, MNRAS, 390, 466

Fischer, J., Sturm, E., González-Alfonso, E., et al. 2010, A\&A, 518, L41

Fisher, J. R., \& Tully, R. B. 1976, A\&A, 53, 397

Genzel, R., Tacconi, L. J., Rigopoulou, D., Lutz, D., \& Tecza, M. 2001, ApJ, 563,527

Greve, A., Neininger, N., Sievers, A., \& Tarchi, A. 2006, A\&A, 459, 441

Greve, A., Neininger, N., Tarchi, A., \& Sievers, A. 2000, A\&A, 364, 409

Groves, B. A., \& Allen, M. G. 2010, NewA, 15, 614

Hailey-Dunsheath, S., Nikola, T., Stacey, G. J., et al. 2010, ApJL, 714, L162

Heckman, T. M., Armus, L., \& Miley, G. K. 1990, ApJS, 74, 833

Herter, T. L., Adams, J. D., De Buizer, J. M., et al. 2012, ApJL, 749, L18

Ho, L. C., Filippenko, A. V., \& Sargent, W. L. W. 1997, ApJS, 112, 315

Hutchings, J. B., Lo, E., Neff, S. G., Stanford, S. A., \& Unger, S. W. 1990, AJ, 100,60

Inui, T., Matsumoto, H., Tsuru, T. G., et al. 2005, PASJ, 57, 135

Karachentsev, I. D., Karachentseva, V. E., Huchtmeier, W. K., \& Makarov, D. I. 2004, AJ, 127, 2031

Kauffmann, G., Heckman, T. M., Tremonti, C., et al. 2003, MNRAS, 346, 1055

Kaufman, M. J., Wolfire, M. G., Hollenbach, D. J., \& Luhman, M. L. 1999, ApJ, 527,795

Kelz, A., Verheijen, M. A. W., Roth, M. M., et al. 2006, PASP, 118, 129

Kennicutt, R. C., Calzetti, D., Aniano, G., et al. 2011, PASP, 123, 1347

Kewley, L. J., Dopita, M. A., Sutherland, R. S., Heisler, C. A., \& Trevena, J. 2001, ApJ, 556, 121

Kobulnicky, H. A., \& Gebhardt, K. 2000, AJ, 119, 1608

Kormendy, J., Fisher, D. B., Cornell, M. E., \& Bender, R. 2009, ApJS, 182,216
Kormendy, J., \& Sanders, D. B. 1992, ApJL, 390, L53

Kreckel, K., Groves, B., Schinnerer, E., et al. 2013, ApJ, 771, 62

Le Floc'h, E., Papovich, C., Dole, H., et al. 2005, ApJ, 632, 169

Maiolino, R., Gallerani, S., Neri, R., et al. 2012, MNRAS, 425, L66

Malhotra, S., Kaufman, M. J., Hollenbach, D., et al. 2001, ApJ, 561, 766

Martin, C. L. 2005, ApJ, 621, 227

Martig, M., Bournaud, F., Teyssier, R., \& Dekel, A. 2009, ApJ, 707, 250

McCarthy, P. J., van Breugel, W., \& Heckman, T. 1987, AJ, 93, 264

McKeith, C. D., Greve, A., Downes, D., \& Prada, F. 1995, A\&A, 293, 703

Monreal-Ibero, A., Arribas, S., Colina, L., et al. 2010, A\&A, 517, A28

Murphy, T. W., Jr., Armus, L., Matthews, K., et al. 1996, AJ, 111, 1025

Pérez-González, P. G., Rieke, G. H., Egami, E., et al. 2005, ApJ, 630, 82

Petric, A. O., Armus, L., Howell, J., et al. 2011, ApJ, 730, 28

Pettini, M., Shapley, A. E., Steidel, C. C., et al. 2001, ApJ, 554, 981

Pilbratt, G. L., Riedinger, J. R., Passvogel, T., et al. 2010, A\&A, 518, L1

Poglitsch, A., Waelkens, C., Geis, N., et al. 2010, A\&A, 518, L2

Rieke, G. H., Young, E. T., Cadien, J., et al. 2004, Proc. SPIE, 5487, 50

Roth, M. M., Kelz, A., Fechner, T., et al. 2005, PASP, 117, 620

Roussel, H., Wilson, C. D., Vigroux, L., et al. 2010, A\&A, 518, L66

Rubin, K. H. R., Weiner, B. J., Koo, D. C., et al. 2010, ApJ, 719, 1503

Rupke, D. S., Veilleux, S., \& Sanders, D. B. 2005, ApJS, 160, 115

Sanders, D. B., Mazzarella, J. M., Kim, D.-C., Surace, J. A., \& Soifer, B. T. 2003, AJ, 126, 1607

Sanders, D. B., Soifer, B. T., Elias, J. H., et al. 1988, ApJ, 325, 74

Sandin, C., Becker, T., Roth, M. M., et al. 2010, A\&A, 515, A35

Sarzi, M., Falcón-Barroso, J., Davies, R. L., et al. 2006, MNRAS, 366, 1151

Shapley, A. E., Steidel, C. C., Pettini, M., \& Adelberger, K. L. 2003, ApJ, 588,65

Sharp, R. G., \& Bland-Hawthorn, J. 2010, ApJ, 711, 818

Shopbell, P. L., \& Bland-Hawthorn, J. 1998, ApJ, 493, 129

Skibba, R. A., Engelbracht, C. W., Dale, D., et al. 2011, ApJ, 738, 89

Sofia, U. J., Lauroesch, J. T., Meyer, D. M., \& Cartledge, S. I. B. 2004, ApJ, 605,272

Sofia, U. J., Parvathi, V. S., Babu, B. R. S., \& Murthy, J. 2011, AJ, 141, 22

Spoon, H. W. W., Farrah, D., Lebouteiller, V., et al. 2013, ApJ, 775, 127

Steidel, C. C., Shapley, A. E., Pettini, M., et al. 2004, ApJ, 604, 534

Sturm, E., González-Alfonso, E., Veilleux, S., et al. 2011, ApJL, 733, L16

Tamburro, D., Rix, H.-W., Leroy, A. K., et al. 2009, AJ, 137, 4424

Taramopoulos, A., Payne, H., \& Briggs, F. H. 2001, A\&A, 365, 360

Tremonti, C. A., Heckman, T. M., Kauffmann, G., et al. 2004, ApJ, 613, 898

Tsai, A.-L., Matsushita, S., Nakanishi, K., et al. 2009, PASJ, 61, 237

Tully, R. B. 1988, Nearby Galaxies Catalog (Cambridge and New York: Cambridge Univ. Press)

Valdes, F., Gupta, R., Rose, J. A., Singh, H. P., \& Bell, D. J. 2004, ApJS, 152,251

Veilleux, S., Cecil, G., \& Bland-Hawthorn, J. 2005, ARA\&A, 43, 769

Veilleux, S., Meléndez, M., Sturm, E., et al. 2013, ApJ, 776, 27

Walter, F., Weiss, A., \& Scoville, N. 2002, ApJL, 580, L21

Watson, M. G., Stanger, V., \& Griffiths, R. E. 1984, ApJ, 286, 144

Weiner, B. J., Coil, A. L., Prochaska, J. X., et al. 2009, ApJ, 692, 187

Westmoquette, M. S., Smith, L. J., Gallagher, J. S., III, et al. 2009, ApJ, 696, 192

Young, E. T., Becklin, E. E., Marcum, P. M., et al. 2012, ApJL, 749, L17

Young, J. S., Claussen, M. J., Kleinmann, S. G., Rubin, V. C., \& Scoville, N. 1988, ApJL, 331, L81 